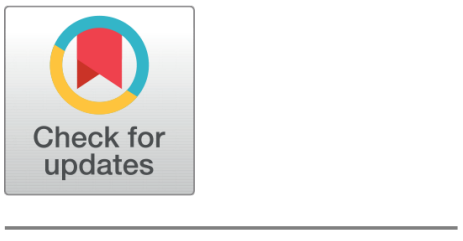

OPEN ACCESS

Received: 19.05.2020

Accepted: 15.09 .2020

Published: 13.10 .2020

Editor: Dr. Natarajan Gajendran

Citation: Saxena P, Singh C, Sharma K (2020) Green design and product stewardship approach for two-warehouse inventory model. Indian Journal of Science and Technology 13(37): 3850-3870. https ://doi.org/10.17485/IJST/v13i37.290

*Corresponding author. anushka.gautam17@gmail.com

Funding: None

Competing Interests: None

Copyright: @ 2020 Saxena et al. This is an open access article distributed under the terms of the Creative Commons Attribution License, which permits unrestricted use, distribution, and reproduction in any medium, provided the original author and source are credited.

Published By Indian Society for Education and Environment (iSee)

ISSN

Print: 0974-6846

Electronic: 0974-5645

\section{Green design and product stewardship approach for two-warehouse inventory model}

\author{
Pratiksha Saxena ${ }^{1}$, Chaman Singh ${ }^{2}$, Kamna Sharma ${ }^{1 *}$ \\ 1 Department of Mathematics, Gautam Buddha University, Greater Noida, Uttar Pradesh, \\ India \\ 2 Department of Mathematics, Acharya Narendra Dev College, University of Delhi, Delhi
}

\section{Abstract}

Background/Objectives: To trim down the recycling cost of any manufactured goods with the help of green design and product stewardship. Methods/Statistical analysis: For the planned EPQ (economic production quantity model) model, all costs are calculated to find total cost and this total cost is optimized with the help of the Hessian matrix. Sensitivity analysis is also carried w.r.t. different parameters, to illustrate the impact of these parameters on the proposed model. The convexity of the total cost function is also checked with the help of mathematical software Mathematica 9.0. Findings: Major finding of the proposed model are as follows: (i) Increase in the number of recycles results in the reduction of the total cost. (ii) Product stewardship parameter has a negative effect on total cost as the PS increases from 1 to 4 units, total cost decreases from 5926.00 to 5918.96 units (see Table 9 ) (similar findings can be written for numeric example 1 after correcting it). (iii) Green design costs have a positive effect on total cost, as the green design cost increases from 3 to 6 units, total cost also increases from 5918.49 to 5920.37 units (see Table 10 ). (iv) increase in the number of recycles results in the reduction of the total cost, as the number of recycles increases from 20 to 50 units total cost decreases from 5922.87 to 5919.12 units (see Table 11 ). Novelty/Applications: The Study of the effects of recycling by this green design and product stewardship approach makes the proposed model distinctive from the existing methods. The proposed model applies to eco-friendly manufacturing items with green design and product stewardship.

Keywords: Green design; product stewardship; production model; own warehouse (OW); rented warehouse (RW); shortage; deterioration

\section{Introduction}

In the current inventory modeling, green design and product stewardship are quite emerging issues. To resolve environmental stumbling blocks, inventory modeling can play a significant role in terms of green design and product stewardship. Manufacturer designs products considering it to be a profitable task, but most 
of the time environment is ignored deliberately or unintentionally. Through green design and product stewardship, a manufacturer can maintain both profit as well as an environmental concern. Frosch and Gallopoulos ${ }^{(1)}$ decided strategies for the manufacturer to reduce environmental impact. To resolve issues of manufacturer, Glantschnig ${ }^{(2)}$ proposed green design and an introduction of issues and challenges related to manufacturing green design. Due to demand of product stewardship, Shapiro and White ${ }^{(3)}$ gave a right start to product stewardship through the life cycle and design of a product. Bellmann and Khare $^{(4)}$ resolved some economic issues in recycling end of life vehicles. To reduce all impact on environment, government should also play a role in safety check on manufacturing process adopted by the manufacturer. In this field, Das ${ }^{(5)}$ revealed the responsibilities of government and manufacture to green concern or environmental issues. Lewis ${ }^{(6)}$ proposed product stewardship in a new pattern and sustainability of it in the Australian packaging industry. A practical approach of putting product stewardship into practice, a commercial moss harvest in North West Oregon studied by Pack and Christy ${ }^{(7)}$. Burger ${ }^{(8)}$ recommended environmental strategies to integrate ecological evolution, remediation, restoration, natural resources damage assessment, and long term stewardship on contaminated lands. Chung and Wee ${ }^{(9)}$ investigated a green component life cycle value on design and reverse manufacturing in a semi-closed supply chain. Natural resources are also used in the manufacturing, and they should be utilized in a smart and economical way, so that Guang et al. ${ }^{(10)}$ proposed a green supply chain based on utilization of natural resources. Christina et al. ${ }^{(11)}$ scrutinized green operation and moderating role of environmental management capability of suppliers manufacturing firm performance. Zhou et al. ${ }^{(12)}$ explored an integrated approach for optimizing green production strategies. Mallidis et al. ${ }^{(13)}$ proposed a design and planning for the green global supply chain under periodic review replenishment policies. Domingo and Rio ${ }^{(14)}$ revealed the linking use stage life cycle inventories with the product design model of usage. Zhu and $\mathrm{He}^{(15)}$ approached green product design in the supply chain under competition. Some of the researchers may approach in a different way to resolve environmental issues like Balakrishnan and Suresh ${ }^{(16)}$. They studied the conceptual framework of multimodal logistics to improve logistics performance and descriptive statistics of multimodal of rail and road transportation. They also analyzed a case study of Ford India's finished vehicle distribution after using a multimodal network. In this same chain. Yadav et al. ${ }^{(17)}$ analyze a sustainable approach for a green supply chain for warehouse with environmental collaboration by using genetic algorithm. To extend yadav et al. ${ }^{(17)}$ model, Khalafi and Zarei ${ }^{(18)}$ proposed a multi-periodic and multiproduct production model considering the environmental issue for the single objective green routing problem with the help of fuzzy theory and mathematical programming. Huo et al. ${ }^{(19)}$ proposed a lean approach by which economic, social and environmental performance of a supply chain can be improve while any green process can improve only environmental performance of supply chain. Recently Du et al. ${ }^{(20)}$ studied strategies for green design in a sustainable supply chain management.

Initially, most of the inventory models were based on the assumption that every manufacturer has its warehouse with unlimited capacity but in a realistic world, it is not possible. Every warehouse has limited capacity due to some real reasons. When manufacturer starts production, he accumulates all well in his warehouse. But when manufacturer got attractive price discount on raw material for bulk purchase or maybe the order cost is too higher than manufacturer have to use RW for the storage purpose. Inventory managers usually attracted to hold more quantity of items that can be stored in an owned warehouse. Rented ware house (RW) may also useful due to its good condition, like low-risk factors, low deterioration rate, and better storage facility, etc. But the manufacturer has to pay some rent or cost which is much greater than own ware house (OW). So that manufacturer uses the RW but tries to utilize the inventory of RW first and then OW inventory. In reality, various factors was induced by the decision-maker to order more items. Recently warehouse situation generally arises when the acquisition is higher than maintaining an RW which have better preservation technology. Hartely ${ }^{(21)}$ was first who proposed a two warehouse inventory system. Sarma ${ }^{(22)}$ generalized Hartely ${ }^{(21)}$ to cover the transportation cost from RW to OW. Dave ${ }^{(23)}$ proposed an economic quantity model with two levels of storage. Kar et al. ${ }^{(24)}$ presented a deterministic inventory model with two levels of storage, a linear trend in demand, and a fixed time horizon. Zohu ${ }^{(25)}$ approaches a multi-warehouse inventory model for deteriorating items with time-varying demand and storage. Yang et al. ${ }^{(26)}$ investigated a two warehouse partial backlogging inventory model for deteriorating items under inflation. To survive in high demand, Singh et al. ${ }^{(27)}$ again presented a two warehouse partial backlogging inventory model for the perishable product having exponential demand. Demand depends on several factors like time etc. to reveal this factor, Lee et al. ${ }^{(28)}$ proposed a two warehouse production model for deteriorating items with time-dependent demand. Singh et al. ${ }^{(29)}$ evaluated an inventory model for deteriorating items with shortages and stock-dependent demand under inflation for two shops under one management. Hariga ${ }^{(30)}$ worked on an inventory model for the multi-warehouse under a fixed and flexible space leasing contract. Sett et al. ${ }^{(31)}$ discussed a two warehouse inventory model with increasing demand and a time-dependent deteriorating rate. Singh et al. ${ }^{(32)}$ proposed an optimal ordering policy for deteriorating items with power form stock dependent demand under two warehouse storage facilities. Kumar et al. ${ }^{(33)}$ developed a two warehouse inventory model for deteriorating items with three component demand rates and a timeproportional backlogging rate and fuzzy environment. Recently, some more researchers like Singh et al. ${ }^{(34)}$ and Khurana ${ }^{(35)}$ 
also presented different approaches for different models.

In this study, a green design and product stewardship approach is used in the model for deteriorating items with shortages. Product stewardship is a predominant factor in green design. It is assumed that the demand rate is a function of price and time. The model is developed with a two-warehouse storage facility. To illustrate the utility of the model, two numerical examples are expounded; convexity and sensitivity analysis is also illuminating the constructive path for the proposed model.

The proposed study is further dealt in the following way: In section 2 deals with research gap analysis regarding the utility of the proposed model, in section 3, notations, and assumptions are provided which is used for the development of the proposed model. In section 4, the problem's definition is presented in the form of a flow chart. In section 5, a mathematical model is derived. In sections 6 and 7, an algorithm to solve the mathematical model and numerical examples are shown respectively.

\section{Research gap}

In the existing literature, different kinds of production inventory models are introduced and studied yet a lot of work had been done by the researcher in the field of green production. In a two warehouses inventory model green design and product stewardship is not used yet. In the proposed paper production depends on demand and still manufacturer needs a rented ware house due to sudden fluctuation in market. To resolve these fluctuation manufacturer should store goods in rented ware house. In the proposed work green design and product stewardship are applicable in the form of cost, which may be responsible to make a product green. This cost may increase the total cost, but it can reduce the recycling cost of inventory and it may reduce the salvage of the system. This concept become the main focal point of this study and that is achieved in it. To show the research gap the previous reports are tabulated as follows ( Table 1 ).

Table 1. Comparison of parameters considered in inventory modeling reported earlier.

\begin{tabular}{|c|c|c|c|c|c|}
\hline \multirow{2}{*}{ References } & \multicolumn{5}{|c|}{ Parameters } \\
\hline & Two warehouse & Recycling & Product Stewardship & Green design & Environmental Management capabilities \\
\hline 1 & No & No & No & No & No \\
\hline 2 & No & No & No & Yes & No \\
\hline 3 & No & Yes & No & No & Yes \\
\hline 4 & No & No & No & No & Yes \\
\hline 5 & No & No & Yes & No & Yes \\
\hline 6 & No & No & Yes & No & Yes \\
\hline 7 & No & No & Yes & No & Yes \\
\hline 8 & No & Yes & No & Yes & No \\
\hline 9 & No & No & No & Yes & Yes \\
\hline 10 & Yes & No & No & No & No \\
\hline 11 & No & Yes & No & No & Yes \\
\hline 12 & No & No & No & Yes & No \\
\hline 13 & No & No & No & Yes & Yes \\
\hline 14 & No & No & No & No & Yes \\
\hline 15 & No & No & No & No & Yes \\
\hline 16 & No & No & No & No & Yes \\
\hline 17 & No & No & No & Yes & No \\
\hline 18 & Yes & No & No & No & Yes \\
\hline 19 & No & No & No & No & Yes \\
\hline 20 & No & No & No & Yes & Yes \\
\hline 21 & Yes & No & No & No & No \\
\hline 22 & Yes & No & No & No & No \\
\hline 23 & Yes & No & No & No & No \\
\hline 24 & Yes & No & No & No & No \\
\hline 25 & No & Yes & No & Yes & No \\
\hline 26 & Yes & No & No & No & No \\
\hline 27 & Yes & No & No & No & No \\
\hline 28 & Yes & No & No & No & No \\
\hline 29 & Yes & No & No & No & No \\
\hline 30 & Yes & No & No & No & No \\
\hline 31 & Yes & No & No & No & No \\
\hline 32 & Yes & No & No & No & No \\
\hline
\end{tabular}

Continued on next page 


\begin{tabular}{llllll}
\hline \multicolumn{2}{c}{ Table 1 continued } & & & & \\
\hline 33 & Yes & No & No & No & No \\
34 & Yes & No & No & No & No \\
35 & Yes & No & No & No & No \\
Proposed & Yes & Yes & Yes & Yes & Yes \\
Model & & & & & \\
\hline
\end{tabular}

\section{Notations and assumptions}

\subsection{Notation}

1. $\mathrm{P}(\mathrm{t})$ Production rate is demand dependent $(\mathrm{KD}(\mathrm{t}))$.

2. $\mathrm{D}(\mathrm{t})$ Demand rate which is $(\mathrm{a}-\mathrm{bp}) \mathrm{t}(\mathrm{a}, \mathrm{b}>0), \mathrm{p}$ is the selling price.

3. $\alpha(t)$ Deterioration rate of inventory items in OW is time-dependent is $\theta_{1} t$.

4. $\beta(t)$ Deterioration rate of inventory items in RW is time-dependent is $\mathrm{i} \theta_{2} t$

5. $I_{1}(t)$ Inventory level in OW at time $\mathrm{t}, \mathrm{t} \in\left[t_{0}, t_{1}\right]$.

6. $I_{2}(t)$ Inventory level in RW at time $\mathrm{t}, \mathrm{t} \in\left[t_{1}, t_{2}\right]$.

7. $I_{3}(t)$ Inventory level in $\mathrm{RW}$ at time $\mathrm{t}, \mathrm{t} \in\left[t_{2}, t_{3}\right]$.

8. $I_{4}(t)$ Inventory level in $\mathrm{OW}$ at time $\mathrm{t}, \mathrm{t} \in\left[t_{3}, t_{4}\right]$.

9. $I_{5}(t)$ Inventory level in OW at time $\mathrm{t}, \mathrm{t} \in\left[t_{1}, t_{3}\right]$.

10. $I_{6}(t)$ Inventory level in $\mathrm{OW}$ at time $\mathrm{t}, \mathrm{t} \in\left[t_{4}, t_{5}\right]$.

11. $I_{7}(t)$ Inventory level in OW at time $t, \mathrm{t} \in\left[t_{5}, t_{6}\right]$.

12. $c_{1}$ Setup cost per production run.

13. $w$ Storage capacity of OW.

14. $c_{2}$ Cost of deteriorated items.

15. $D_{i}$ Quantity of deteriorated items during the production cycle.

16. $H_{i}$ Quantity of stored items during the production cycle in OW and RW.

17. $C_{O W}$ Carrying cost per inventory unit per unit time in OW.

18. $C_{R W}$ Carrying cost per inventory unit per unit time in RW.

19. $\mathrm{G}_{d}$ Green design life cycle cost.

20. $\mathrm{G}_{d v}$ Fixed product life cycle design cost ratio for green design.

21. N Number of life cycles before a product is disposed of or recycles.

22. RW Rented ware house.

23. OW Own ware house.

24. $\mathrm{R}_{D V}$ Variable product life design cost ratio for green design.

25. $\mathrm{R}_{j}$ Reliability of the function.

26. PS Product stewardship.

27. TC Total cost.

\subsection{Assumption}

1. Demand is price and time dependent in this model. $\mathrm{D}(\mathrm{t})=(\mathrm{a}-\mathrm{bp}) \mathrm{t}$, where $\mathrm{a}, \mathrm{b}$ are constant, $\mathrm{p}$ is selling price of the commodity and $t$ is time. (for example Zhou ${ }^{(25)}$ and Singh et. al ${ }^{(27)}$ )

2. The OW has a limited capacity of $w$ units and the RW has unlimited capacity.

3. The inventory cost of RW is greater than OW.

4. Inventory decreased due to demand and deterioration only.

5. Production rate depends on time and rate of production is $\mathrm{KD}(\mathrm{t})$, where $\mathrm{K}$ is constant.

6. The deteriorating rate depends on time and deteriorating units cannot be replaced or repaired. Deterioration rate for the model is $\theta t$, where $\theta$ is deterioration rate and $\mathrm{t}$ in time.(for example sett et. al ${ }^{(31)}$ )

7. The RW is located near the OW so the transportation cost is negligible.

8. Deterioration rate of RW is lower than the OW.

9. The lead time is considered as negligible.

10. The shortage is allowed and completely backlogged. 

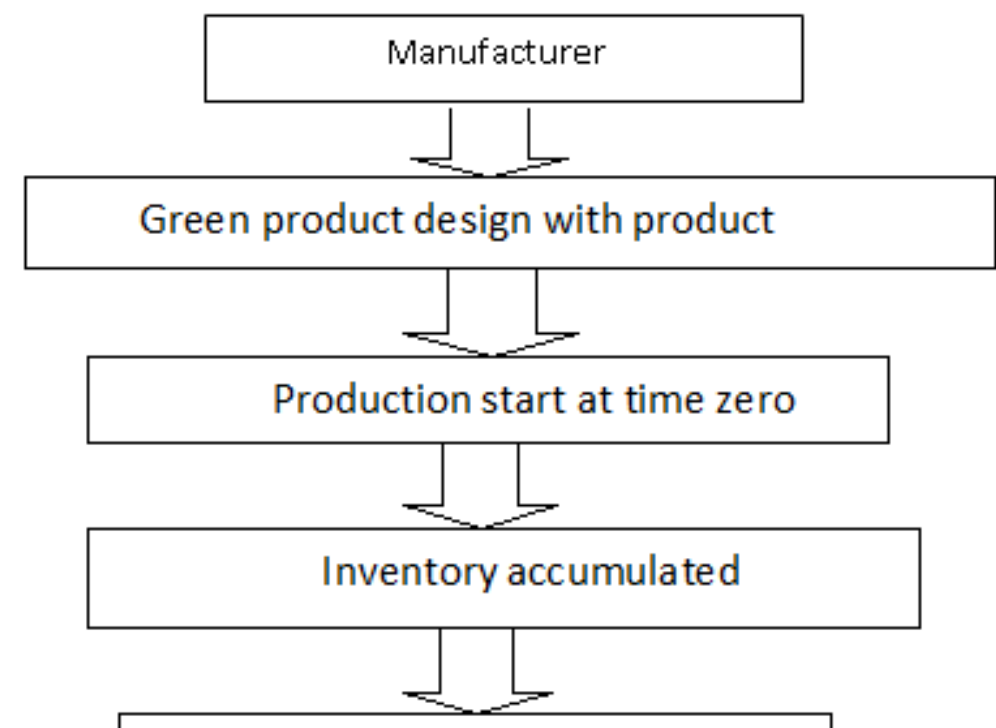

Inventory stores in OW

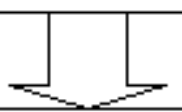

Residue inventory stored in RW

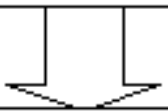

Inventory depleted due to deterioration and demand

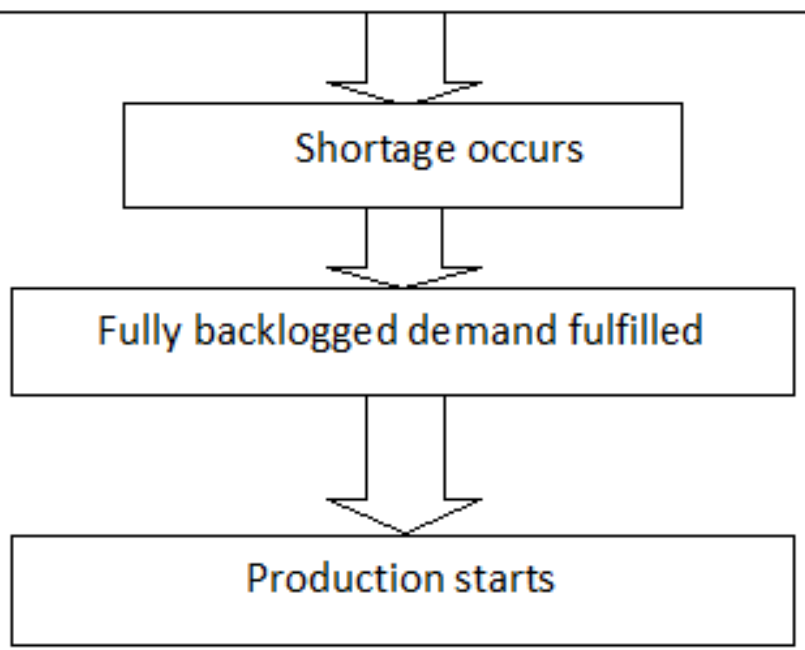

Fig 1. Flow chart of the production model 


\section{Mathematical model}

In the proposed mathematical model, price and time-dependent demand are considered for different inventory levels. Here the production starts at zero time. Figure 1 shows the different inventory levels during the production cycle when two different warehouses are used OW and RW.

Throughout the time interval, 0 to $t_{1}$ inventory level levitate at the rate of $P-D(t)$ for manufactured own warehouse, where $P$ is the rate of production, and $\mathrm{D}(\mathrm{t})$ is the demand rate. This situation solved with the help of differential equation

$$
\frac{d I_{1}(t)}{d t}+\alpha(t) I_{1}(t)=P-D(t)
$$

With the boundary conditions $0 \leq t \leq t_{1}, \quad I_{1}(0)=0$

Throughout the time interval, $\mathrm{t}_{1}$ to $\mathrm{t}_{2}$ inventory level levitate at the rate of $P-D(t)$ for the rented warehouse, where $\mathrm{P}$ is the rate of production, and $\mathrm{D}(\mathrm{t})$ is the demand rate. This situation solved with the help of differential equation

$$
\frac{d I_{2}(t)}{d t}+\beta(t) I_{2}(t)=P-D(t)
$$

With the boundary conditions $t_{1} \leq t \leq t_{2}, \quad I_{2}\left(t_{1}\right)=0$

Throughout the time interval, $\mathrm{t}_{2}$ to $t_{3}$ inventory level diminished at the rate of $D(t)$ for the rented warehouse, where $\mathrm{D}(\mathrm{t})$ is the demand rate. This situation is solved with the help of differential equation

$$
\frac{d I_{3}(t)}{d t}+\beta(t) I_{3}(t)=-D(t)
$$

With the boundary conditions $t_{2} \leq t \leq t_{3}, \quad I_{3}\left(t_{3}\right)=0$

Throughout the time interval, $\mathrm{t}_{3}$ to $t_{4}$ inventory level diminished at the rate of $D(t)$ for manufactured own warehouse, where $\mathrm{D}(\mathrm{t})$ is the demand rate. This situation is solved with the help of differential equation

$$
\frac{d I_{4}(t)}{d t}+\alpha(t) I_{4}(t)=-D(t)
$$

With the boundary conditions $t_{3} \leq t \leq t_{4}, \quad I_{4}\left(t_{4}\right)=0$

Throughout the time interval, $\mathrm{t}_{1}$ to $\mathrm{t}_{3}$ inventory level diminished at the rate of $\alpha(t)$ from manufactured own warehouse, where $\alpha(t)$ is the deterioration rate of own warehouse. In time interval $t_{1}$ to $t_{3}$ inventory stored in the manufactured own warehouse deteriorate. This deteriorated inventory is calculated with the help of differential equations given below, where ' $w$ ' is the capacity of manufactured own warehouse.

$$
\frac{d I_{5}(t)}{d t}+\alpha(t) I_{5}(t)=0
$$

With the boundary conditions $t_{1} \leq t \leq t_{3}, \quad I_{5}\left(t_{1}\right)=w$

Throughout the time interval, $\mathrm{t}_{4}$ to $\mathrm{t}_{5}$ shortage occurs at the rate of $D(t)$, where $\mathrm{D}(\mathrm{t})$ is the demand rate. This $\mathrm{I}_{6}(\mathrm{t})$ inventory is calculated with the help of differential equation

$$
\frac{d I_{6}(t)}{d t}=-D(t)
$$

With the boundary conditions $t_{4} \leq t \leq t_{5}, \quad I_{6}\left(t_{4}\right)=0$

Throughout the time interval $\mathrm{t}_{5}$ to $\mathrm{t}_{6}$ production starts again and inventory level increases at the rate $P-D(t)$, where $\mathrm{P}$ and $\mathrm{D}(\mathrm{t})$ are the rates of production the rate of demand respectively. The levitated inventory is calculated with the help of differential equation

$$
\frac{d I_{7}(t)}{d t}+\alpha(t) I_{4}(t)=P-D(t)
$$

With the boundary conditions $t_{5} \leq t \leq t_{6}, \quad I_{7}\left(t_{6}\right)=0$ 
On solving above differential equation (1), subject to the boundary condition

$$
I_{1}(t)=\left\{(1-k)(a-b p)\left[\frac{t^{2}}{2}-\frac{3 \theta_{1} t^{4}}{8}-\frac{\theta_{1}^{2} t^{6}}{16}\right]\right\}
$$

On solving above differential equation (2), subject to the boundary condition

$$
I_{2}(t)=\left\{(1-k)(a-b p)\left(1-\frac{\theta_{2}^{2} t_{1}^{4}}{2}\right)\left[\left(\frac{t^{2}}{2}-\frac{\theta_{2} t^{4}}{8}\right)-\left(\frac{\theta_{2} t^{4}}{8}+\frac{t_{1}^{2}}{2}\right)\right]\right\}
$$

On solving above differential equation (3), subject to the boundary condition

$$
I_{3}(t)=\left\{(a-b p)\left[\left(\frac{\theta_{2} t^{2}}{2}-1\right)\left(\frac{\theta_{2} t_{3}^{4}}{8}+\frac{t_{3}^{2}}{2}\right)-\left(\frac{\theta_{2} t^{4}}{4}-\frac{t^{2}}{2}-\frac{\theta_{2} t^{4}}{8}+\frac{\theta_{2}^{2} t^{6}}{8}\right)\right]\right\}
$$

On solving above differential equation (4), subject to the boundary condition

$$
I_{4}(t)=\left\{(a-b p)\left[\left(\frac{\theta_{1} t^{2}}{2}-1\right)\left(\frac{\theta_{1} t_{4}^{4}}{8}+\frac{t_{4}^{2}}{2}\right)-\left(\frac{\theta_{1} t^{4}}{4}-\frac{t^{2}}{2}-\frac{\theta_{1} t^{4}}{8}+\frac{\theta_{1}^{2} t^{6}}{8}\right)\right]\right\}
$$

On solving above differential equation (5), subject to the boundary condition

$$
I_{5}(t)=w\left(1-\frac{\theta_{1} t^{2}}{2}\right)\left(1+\frac{\theta_{1} t_{1}^{2}}{2}\right)
$$

On solving above differential equation (6), subject to the boundary condition

$$
I_{6}(t)=(a-b p)\left[\left(\frac{t_{4}^{2}}{2}-\frac{t^{2}}{2}\right)\right]
$$

On solving above differential equation (7), subject to the boundary condition

$$
I_{7}(t)=\left\{(1-k)(a-b p)\left(1-\frac{\theta_{1} t^{2}}{2}\right)\left[\left(\left(\frac{t^{2}}{2}-\frac{\theta_{1} t^{4}}{8}\right)\right)-\left(\frac{\theta_{1} t_{6}^{4}}{8}+\frac{t_{6}^{2}}{2}\right)\right]\right\}
$$

The different inventory levels obtained as follows:

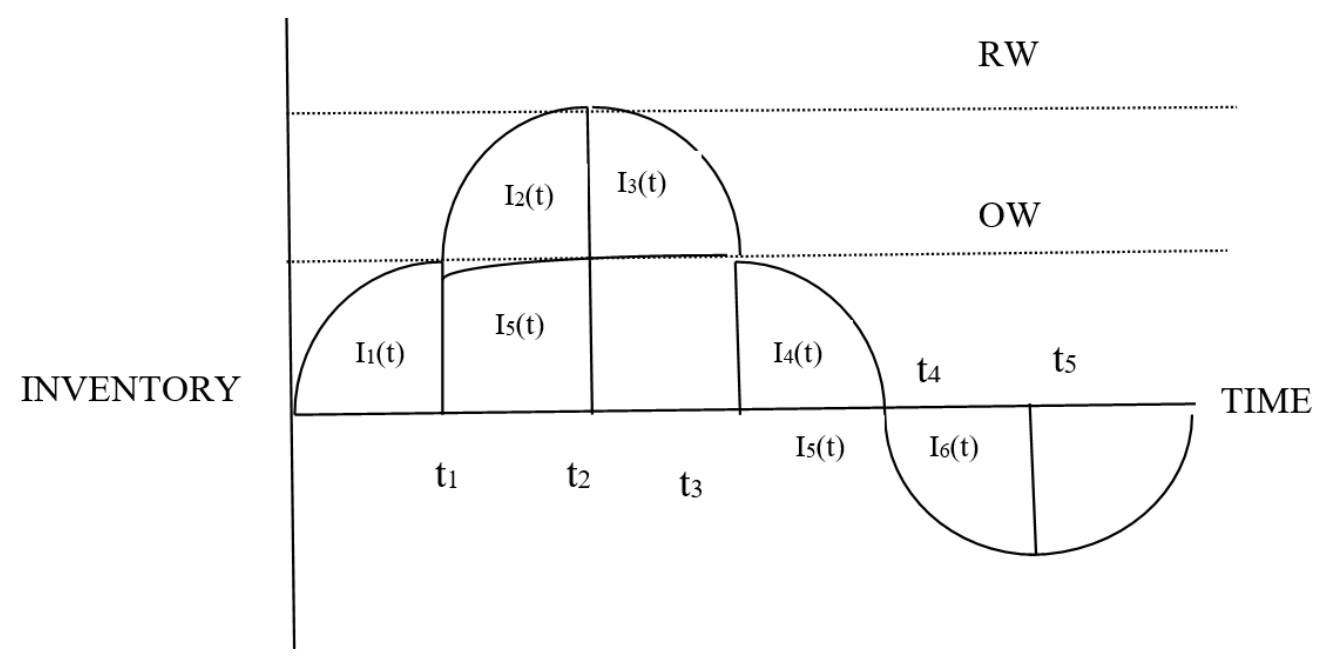

Fig 2. Inventory level 
To find out the relation between $t_{2}, t_{3}$, and $t_{5}$ the equation of continuity is solved

$$
\begin{gathered}
I_{2}\left(t_{2}\right)=I_{3}\left(t_{2}\right)\left\{(1-k)(a-b p)\left(1-\frac{\theta_{2}^{2} t_{1}^{4}}{2}\right)\left[\left(\frac{t_{2}^{2}}{2}-\frac{\theta_{2} t_{2}^{4}}{8}\right)-\left(\frac{\theta_{2} t_{1}^{4}}{8}+\frac{t_{1}^{2}}{2}\right)\right]\right\}= \\
\left\{(a-b p)\left[\left(\frac{\theta_{2} t_{2}^{2}}{2}-1\right)\left(\frac{\theta_{2} t_{3}^{4}}{8}+\frac{t_{3}^{2}}{2}\right)-\left(\frac{\theta_{2} t_{2}^{4}}{4}-\frac{t_{2}^{2}}{2}-\frac{\theta_{2} t_{2}^{4}}{8}+\frac{\theta_{2}^{2} t_{2} s^{6}}{8}\right)\right]\right\} \\
I_{4}\left(t_{3}\right)=I_{5}\left(t_{3}\right)\left(\frac{t_{4}^{2}}{2}\right)+\left(\frac{\theta_{2} t_{4}^{4}}{8}\right)+\frac{w\left(1-\frac{\theta_{1} t_{1}^{2}}{2}\right)}{(a-b p)}-\left(\frac{t_{3}^{2}}{2}\right)+\left(\frac{\theta_{2} t_{3}^{4}}{8}\right)=0 \\
\left.I_{7}\left(t_{5}\right)=-I_{6}\left(t_{5}\right)\left\{(1-k)(a-b p)\left(1-\frac{\theta_{1} t^{2}}{2}\right)\left[\left(\frac{t_{5}^{2}}{2}-\frac{\theta_{1} t_{5}^{4}}{8}\right)\right)-\left(\frac{\theta_{1} t_{6}^{4}}{8}+\frac{t_{6}^{2}}{2}\right)\right]\right\}=-(a-b p)\left(\frac{t_{4}^{2}}{2}-\frac{t_{5}^{2}}{2}\right)
\end{gathered}
$$

Inventory stored at the manufacturer own warehouse is calculated by integrating different inventory level at a different time period

$$
\begin{gathered}
I_{O W}(t)=\int_{0}^{t_{1}} I_{1}(t) d t+\int_{t_{3}}^{t_{4}} I_{4}(t) d t+\int_{t_{1}}^{t_{3}} I_{5}(t) d t+\int_{t_{4}}^{t_{5}} I_{6}(t) d t+\int_{t_{5}}^{t_{6}} I_{7}(t) d t \\
I_{O W}(t)=(a-b p)(1-k)\left[\frac{t_{1}^{2}}{2}+\frac{3 \theta_{1} t_{1}^{4}}{8}-\frac{\theta_{1}^{2} t_{1}^{6}}{16}\right]+ \\
(a-b p)\left[\left(\frac{t_{4}^{2}}{2}+\frac{\theta_{1} t_{4}^{2}}{2}\right)\left(\frac{\theta_{1} t_{4}^{3}}{6}-\frac{\theta_{1} t_{3}^{3}}{6}-t_{4}+t_{3}\right)-\left(\frac{\theta_{1} t_{4}^{5}}{20}-\frac{\theta_{1} t_{3}^{5}}{20}+\frac{\theta_{1}^{2} t_{4}^{7}}{56}-\frac{\theta_{1}^{2} t_{3}^{7}}{56}-\frac{t_{4}^{3}}{6}+\frac{t_{3}^{3}}{6}-\frac{\theta_{2} t_{4}^{5}}{40}+\frac{\theta_{2} t_{3}^{5}}{40}\right)\right]- \\
{\left[w\left(1+\frac{\theta_{1} t_{1}^{2}}{2}\right)\left(t_{3}-t_{1}-\frac{\theta_{1} t_{3}^{3}}{6}+\frac{\theta_{1} t_{1}^{3}}{6}\right)\right]+(1-k)(a-b p)} \\
{\left[\frac{t_{6}^{3}}{6}+\frac{t_{5}^{3}}{6}-\frac{3 \theta_{1} t_{6}^{5}}{40}+\frac{\theta_{1} t_{5}^{5}}{40}-\frac{\theta_{1}^{2} t_{6}^{7}}{112}+\frac{\theta_{1}^{2} t_{5}^{7}}{112}-\left(\frac{t_{6}^{2}}{2}+\frac{\theta_{1} t_{6}^{4}}{8}\right)\left(t_{6}-t_{5}-\frac{\theta_{1} t_{6}^{3}}{6}+\frac{\theta_{1} t_{5}^{3}}{6}\right)\right]+(a-b p)\left[\frac{t_{4}^{2}}{2}\left(t_{5}-t_{4}\right)-\frac{t_{5}^{3}}{6}+\frac{t_{4}^{3}}{6}\right]}
\end{gathered}
$$

The inventory stored at the rented warehouse when the capacity of own warehouse is filled can be calculated as given below:

$$
\begin{gathered}
I_{R W}(t)=\int_{t_{1}}^{t_{2}} I_{2}(t) d t+\int_{t_{2}}^{t_{3}} I_{3}(t) d t \\
\mathrm{I}_{\mathrm{RW}}(\mathrm{t})=(1-\mathrm{k})(\mathrm{a}-\mathrm{bp})\left[\left(\frac{\mathrm{t}_{2}^{3}}{6}-\frac{\mathrm{t}_{1}^{3}}{6}+\frac{3 \theta_{2} \mathrm{t}_{2}^{5}}{40}-\frac{3 \theta_{2} \mathrm{t}_{1}^{5}}{40}-\frac{\theta_{2}^{2} \mathrm{t}_{2}^{7}}{112}+\frac{\theta_{2}^{2} \mathrm{t}_{1}^{7}}{112}\right)-\left(\frac{\theta_{2} t_{1}^{4}}{8}+\frac{t_{1}^{2}}{2}\right)\left(t_{2}-t_{1}-\frac{\theta_{2} \mathrm{t}_{2}^{3}}{6}-\frac{\theta_{2} \mathrm{t}_{1}^{3}}{6}\right)\right]+ \\
(\mathrm{a}-\mathrm{bp})\left[\left(\frac{\theta_{2} \mathrm{t}_{3}^{4}}{2}+\frac{\mathrm{t}_{3}^{3}}{8}\right)\left(\frac{\theta_{2} \mathrm{t}_{3}^{3}}{6}-\frac{\theta_{2} \mathrm{t}_{2}^{3}}{6}-\mathrm{t}_{3}+\mathrm{t}_{2}\right)-\left(\frac{\theta_{2} \mathrm{t}_{3}^{5}}{20}-\frac{\theta_{2} \mathrm{t}_{2}^{5}}{20}+\frac{\theta_{2}{ }^{2} \mathrm{t}_{3}^{5}}{56}-\frac{\theta_{2}^{2} \mathrm{t}_{2}^{5}}{56}-\frac{\mathrm{t}_{3}^{3}}{6}+\frac{\mathrm{t}_{2}^{3}}{6}-\frac{\theta_{2} \mathrm{t}_{3}^{5}}{40}+\frac{\theta_{2} \mathrm{t}_{2}^{5}}{40}\right)\right]
\end{gathered}
$$


The number of deteriorating stocks in both own and rented warehouse during the production cycle:

$$
\begin{aligned}
& D_{i}=\int_{t_{0}}^{t_{1}} \alpha(t) I_{1}(t) d t+\int_{t_{1}}^{t_{3}} \alpha(t) I_{5}(t) d t+\int_{t_{1}}^{t_{2}} \beta(t) I_{2}(t) d t+\int_{t_{2}}^{t_{3}} \beta(t) I_{3}(t) d t+\int_{t_{3}}^{t_{4}} \alpha(t) I_{4}(t) d t= \\
& (1-\mathrm{k})(\mathrm{a}-\mathrm{bp}) \theta_{1}\left\{\frac{\mathrm{t}_{1}^{4}}{6}-\frac{3 \theta_{1} \mathrm{t}_{1}^{8}}{64}+\frac{\theta_{1}^{2} \mathrm{t}_{1}^{8}}{112}\right\}+(1-\mathrm{k})(\mathrm{a}-\mathrm{bp}) \\
& {\left[\left(\frac{\mathrm{t}_{2}^{3}}{8}+\frac{\mathrm{t}_{1}^{3}}{8}-\frac{\theta_{1} \mathrm{t}_{2}^{5}}{40}+\frac{\theta_{1} \mathrm{t}_{1}^{5}}{40}-\frac{\theta_{1}^{2} \mathrm{t}_{2}^{8}}{128}+\frac{\theta_{1}^{2} \mathrm{t}_{1}^{8}}{128}-\left(\frac{t_{1}^{2}}{2}+\frac{\theta_{1} t_{1}^{4}}{8}\right)\left(t_{2}-t_{1}-\frac{\theta_{1} t_{2}^{3}}{6}+\frac{\theta_{1} t_{1}^{3}}{6}\right)\right]+\right.} \\
& w \theta_{1}\left(1+\frac{\theta_{1} t_{1}^{2}}{2}\right)\left(\frac{\theta_{1} t_{1}^{4}}{8}-\frac{\theta_{1} t_{3}^{4}}{8}+\frac{t_{3}^{2}}{2}-\frac{t_{1}^{2}}{2}\right)+(a-b p) \theta_{2}\left\{\left(\frac{\theta_{2} t_{3}^{4}}{8}-\frac{\theta_{2} \mathrm{t}_{2}^{4}}{8}+\frac{\mathrm{t}_{3}^{2}}{2}-\frac{\mathrm{t}_{2}^{2}}{2}\right)\right. \\
& \left.\left.\left(\frac{\theta_{2} \mathrm{t}_{3}^{4}}{4}+\frac{\mathrm{t}_{3}^{2}}{2}\right)-\left(\frac{\theta_{2} \mathrm{t}_{3}^{6}}{24}-\frac{\theta_{2} \mathrm{t}_{2}^{6}}{24}+\frac{\theta_{2} \mathrm{t}_{2}^{6}}{48}-\frac{\theta_{2} \mathrm{t}_{3}^{6}}{48}-\frac{\theta_{2} \mathrm{t}_{2}^{8}}{64}+\frac{\theta_{2} \mathrm{t}_{3}^{8}}{64}+\frac{\mathrm{t}_{2}^{4}}{8}-\frac{\mathrm{t}_{3}^{4}}{8}\right)\right\}+\frac{\theta^{2}}{84}\right) \\
& (\mathrm{a}-b p) \theta_{1}\left\{\left(\frac{\theta_{1} \mathrm{t}_{4}^{4}}{8}-\frac{\theta_{1} \mathrm{t}_{3}^{4}}{8}+\frac{\mathrm{t}_{3}^{2}}{2}-\frac{\mathrm{t}_{4}^{2}}{2}\right)\left(\frac{\theta_{1} \mathrm{t}_{4}^{4}}{4}+\frac{\mathrm{t}_{4}^{2}}{2}\right)-\left(\frac{\theta_{1} \mathrm{t}_{4}^{6}}{24}-\frac{\theta_{1} \mathrm{t}_{3}^{6}}{24}+\frac{\theta_{1} \mathrm{t}_{3}^{6}}{48}-\frac{\theta_{1} \mathrm{t}_{4}^{6}}{48}-\frac{\theta_{1}^{2} \mathrm{t}_{1}^{8}}{64}+\frac{\theta_{1}^{2} \mathrm{t}_{4}^{8}}{64}+\frac{\mathrm{t}_{3}^{4}}{8}-\frac{\mathrm{t}_{4}^{4}}{8}\right)\right\}+ \\
& (1-\mathrm{k})(\mathrm{a}-\mathrm{bp})\left[\left(\frac{\mathrm{t}_{6}^{3}}{8}+\frac{\mathrm{t}_{5}^{3}}{8}-\frac{\theta_{1} \mathrm{t}_{6}^{5}}{40}+\frac{\theta_{1} \mathrm{t}_{5}^{5}}{40}-\frac{\theta_{1}^{2} \mathrm{t}_{6}^{8}}{128}+\frac{\theta_{1}^{2} \mathrm{t}_{5}^{8}}{128}-\left(\frac{\mathrm{t}_{6}^{2}}{2}+\frac{\theta_{1} \mathrm{t}_{6}^{4}}{8}\right)\left(\mathrm{t}_{6}-\mathrm{t}_{5}-\frac{\theta_{1} \mathrm{t}_{6}^{3}}{6}+\frac{\theta_{1} \mathrm{t}_{5}^{3}}{6}\right)\right]\right.
\end{aligned}
$$

Total holding cost for rented and own warehouse:

$$
\begin{aligned}
& H_{i}=\int_{t_{0}}^{t_{1}} \alpha(t) I_{1}(t) d t+\int_{t_{1}}^{t_{3}} \alpha(t) I_{5}(t) d t+\int_{t_{1}}^{t_{2}} \beta(t) I_{2}(t) d t+\int_{t_{2}}^{t_{3}} \beta(t) I_{3}(t) d t+\int_{t_{3}}^{t_{4}} \alpha(t) I_{4}(t) d t \\
& =(1-\mathrm{k})(\mathrm{a}-\mathrm{bp}) \theta_{1}\left\{\frac{\mathrm{t}_{1}^{4}}{6}-\frac{3 \theta_{1} \mathrm{t}_{1}^{8}}{64}+\frac{\theta_{1}^{2} \mathrm{t}_{1}^{8}}{112}\right\}+(1-\mathrm{k})(\mathrm{a}-\mathrm{bp})\left[\left(\frac{\mathrm{t}_{2}^{3}}{8}+\frac{\mathrm{t}_{1}^{3}}{8}-\frac{\theta_{1} \mathrm{t}_{2}^{5}}{40}+\frac{\theta_{1} \mathrm{t}_{1}^{5}}{40}-\frac{\theta_{1}^{2} \mathrm{t}_{2}^{8}}{128}+\right.\right. \\
& \left.\quad \frac{\theta_{1}^{2} t_{1}^{8}}{128}-\left(\frac{t_{1}^{2}}{2}+\frac{\theta_{1} t_{1}^{4}}{8}\right)\left(t_{2}-t_{1}-\frac{\theta_{1} t_{2}^{3}}{6}+\frac{\theta_{1} t_{1}^{3}}{6}\right)\right]+w \theta_{1}\left(1+\frac{\theta_{1} t_{1}^{2}}{2}\right)\left(\frac{\theta_{1} t_{1}^{4}}{8}-\frac{\theta_{1} t_{3}^{4}}{8}+\frac{t_{3}^{2}}{2}-\frac{t_{1}^{2}}{2}\right)+ \\
& (a-\mathrm{bp}) \theta_{2}\left\{\left(\frac{\theta_{2} t_{3}^{4}}{8}-\frac{\theta_{2} t_{2}^{4}}{8}+\frac{t_{3}^{2}}{2}-\frac{t_{2}^{2}}{2}\right)\left(\frac{\theta_{2} t_{3}^{4}}{4}+\frac{t_{3}^{2}}{2}\right)-\left(\frac{\theta_{2} t_{3}^{6}}{24}-\frac{\theta_{2} t_{2}^{6}}{24}+\frac{\theta_{2} t_{2}^{6}}{48}-\frac{\theta_{2} t_{3}^{6}}{48}-\frac{\theta_{2} t_{2}^{8}}{64}+\frac{\theta_{2} t_{3}^{8}}{64}+\frac{t_{2}^{4}}{8}-\frac{t_{3}^{4}}{8}\right)\right\}+ \\
& (a-b p) \theta_{1}\left\{\left(\frac{\theta_{1} t_{4}^{4}}{8}-\frac{\theta_{1} t_{3}^{4}}{8}+\frac{t_{3}^{2}}{2}-\frac{t_{4}^{2}}{2}\right)\left(\frac{\theta_{1} t_{4}^{4}}{4}+\frac{t_{4}^{2}}{2}\right)-\left(\frac{\theta_{1} t_{4}^{6}}{24}-\frac{\theta_{1} t_{3}^{6}}{24}+\frac{\theta_{1} t_{3}^{6}}{48}-\frac{\theta_{1} t_{4}^{6}}{48}-\frac{\theta_{1}^{2} t_{1}^{8}}{64}+\frac{\theta_{1}^{2} t_{4}^{8}}{64}+\frac{t_{3}^{4}}{8}-\frac{t_{4}^{4}}{8}\right)\right\}+ \\
& (1-k)(a-b p)\left[\left(\frac{t_{6}^{3}}{8}+\frac{t_{5}^{3}}{8}-\frac{\theta_{1} t_{6}^{5}}{40}+\frac{\theta_{1} t_{5}^{5}}{40}-\frac{\theta_{1}^{2} t_{6}^{8}}{128}+\frac{\theta_{1}^{2} t_{5}^{8}}{128}-\left(\frac{t_{6}^{2}}{2}+\frac{\theta_{1} t_{6}^{4}}{8}\right)\left(t_{6}-t_{5}-\frac{\theta_{1} t_{6}^{3}}{6}+\frac{\theta_{1} t_{5}^{3}}{6}\right)\right]\right.
\end{aligned}
$$

Shortage cost: When a shortage occurs in any production system manufacturer lose some sale. This cost is called a shortage cost.

$$
=-C_{s} \int_{t_{4}}^{t_{5}} I_{6}(t) d t=C_{s}(a-b p)\left\{\left(\frac{t_{5}^{3}}{6}-\frac{t_{4}^{3}}{6}-\frac{t_{4}^{2}}{2}\left(t_{5}-t_{4}\right)\right)\right\}
$$

Green design cost: When a green design product is manufactured there is some extra expenditure that occurs in this process, that expenditure is called green design cost.

$$
G(N)=\frac{G_{D}}{P S}\left\{\frac{G_{D F}}{N}+N \cdot R_{D V} \cdot \prod_{j=1}^{2} R_{j}\right\}
$$


$\mathrm{TC}=$ Setup cost + Holding cost + Deterioration cost + Shortage cost + Green design cost

$$
\begin{aligned}
& \mathrm{TC}=\mathrm{A}+(1-\mathrm{k})(\mathrm{a}-\mathrm{bp}) \theta_{1}\left\{\frac{\mathrm{t}_{1}^{4}}{6}-\frac{3 \theta_{1} \mathrm{t}_{1}^{8}}{64}+\frac{\theta_{1}^{2} \mathrm{t}_{1}^{8}}{112}\right\}+(1-\mathrm{k})(\mathrm{a}-\mathrm{bp})\left[\left(\frac{\mathrm{t}_{2}^{3}}{8}+\frac{\mathrm{t}_{1}^{3}}{8}-\frac{\theta_{1} \mathrm{t}_{2}^{5}}{40}+\frac{\theta_{1} \mathrm{t}_{1}^{5}}{40}-\frac{\theta_{1}^{2} \mathrm{t}_{2}^{8}}{128}+\right.\right. \\
& \left.\frac{\theta_{1}^{2} t_{1}^{8}}{128}-\left(\frac{t_{1}^{2}}{2}+\frac{\theta_{1} t_{1}^{4}}{8}\right)\left(t_{2}-t_{1}-\frac{\theta_{1} t_{2}^{3}}{6}+\frac{\theta_{1} t_{1}^{3}}{6}\right)\right]+w \theta_{1}\left(1+\frac{\theta_{1} t_{1}^{2}}{2}\right)\left(\frac{\theta_{1} t_{1}^{4}}{8}-\frac{\theta_{1} t_{3}^{4}}{8}+\frac{t_{3}^{2}}{2}-\frac{t_{1}^{2}}{2}\right)+(a- \\
& \text { bр) } \theta_{2}\left\{\left(\frac{\theta_{2} \mathrm{t}_{3}^{4}}{8}-\frac{\theta_{2} \mathrm{t}_{2}^{4}}{8}+\frac{\mathrm{t}_{3}^{2}}{2}-\frac{\mathrm{t}_{2}^{2}}{2}\right)\left(\frac{\theta_{2} \mathrm{t}_{3}^{4}}{4}+\frac{\mathrm{t}_{3}^{2}}{2}\right)-\left(\frac{\theta_{2} \mathrm{t}_{3}^{6}}{24}-\frac{\theta_{2} \mathrm{t}_{2}^{6}}{24}+\frac{\theta_{2} \mathrm{t}_{2}^{6}}{48}-\frac{\theta_{2} \mathrm{t}_{3}^{6}}{48}-\frac{\theta_{2} \mathrm{t}_{2}^{8}}{64}+\frac{\theta_{2} \mathrm{t}_{3}^{8}}{64}+\frac{\mathrm{t}_{2}^{4}}{8}-\frac{\mathrm{t}_{3}^{4}}{8}\right)\right\}+ \\
& (a-b p) \theta_{1}\left\{\left(\frac{\theta_{1} t_{4}^{4}}{8}-\frac{\theta_{1} t_{3}^{4}}{8}+\frac{t_{3}^{2}}{2}-\frac{t_{4}^{2}}{2}\right)\left(\frac{\theta_{1} t_{4}^{4}}{4}+\frac{t_{4}^{2}}{2}\right)-\left(\frac{\theta_{1} t_{4}^{6}}{24}-\frac{\theta_{1} t_{3}^{6}}{24}+\frac{\theta_{1} t_{3}^{6}}{48}-\frac{\theta_{1} t_{4}^{6}}{48}-\frac{\theta_{1}^{2} t_{1}^{8}}{64}+\frac{\theta_{1}^{2} t_{4}^{8}}{64}+\frac{t_{3}^{4}}{8}-\right.\right. \\
& \left.\left.\frac{t_{4}^{4}}{8}\right)\right\}+(1-k)(a-b p)\left[\left(\frac{t_{6}^{3}}{8}+\frac{t_{5}^{3}}{8}-\frac{\theta_{1} t_{6}^{5}}{40}+\frac{\theta_{1} t_{5}^{5}}{40}-\frac{\theta_{1}^{2} t_{6}^{8}}{128}+\frac{\theta_{1}^{2} t_{5}^{8}}{128}-\left(\frac{t_{6}^{2}}{2}+\frac{\theta_{1} t_{6}^{4}}{8}\right)\left(t_{6}-t_{5}-\frac{\theta_{1} t_{6}^{3}}{6}+\frac{\theta_{1} t_{5}^{3}}{6}\right)\right]\right. \\
& \left.\left(\frac{t_{1}^{2}}{2}+\frac{\theta_{1} t_{1}^{4}}{8}\right)\left(t_{2}-t_{1}-\frac{\theta_{1} t_{2}^{3}}{6}+\frac{\theta_{1} t_{1}^{3}}{6}\right)\right]+w \theta_{1}\left(1+\frac{\theta_{1} t_{1}^{2}}{2}\right)\left(\frac{\theta_{1} t_{1}^{4}}{8}-\frac{\theta_{1} t_{3}^{4}}{8}+\frac{t_{3}^{2}}{2}-\frac{t_{1}^{2}}{2}\right)+(a-b p) \theta_{2}\left\{\left(\frac{\theta_{2} t_{3}^{4}}{8}-\right.\right. \\
& \left.\left.\frac{\theta_{2} \mathrm{t}_{2}^{4}}{8}+\frac{\mathrm{t}_{3}^{2}}{2}-\frac{\mathrm{t}_{2}^{2}}{2}\right)\left(\frac{\theta_{2} \mathrm{t}_{3}^{4}}{4}+\frac{\mathrm{t}_{3}^{2}}{2}\right)-\left(\frac{\theta_{2} \mathrm{t}_{3}^{6}}{24}-\frac{\theta_{2} \mathrm{t}_{2}^{6}}{24}+\frac{\theta_{2} \mathrm{t}_{2}^{6}}{48}-\frac{\theta_{2} \mathrm{t}_{3}^{6}}{48}-\frac{\theta_{2} \mathrm{t}_{2}^{8}}{64}+\frac{\theta_{2} \mathrm{t}_{3}^{8}}{64}+\frac{\mathrm{t}_{2}^{4}}{8}-\frac{\mathrm{t}_{3}^{4}}{8}\right)\right\}+(\mathrm{a}- \\
& \text { bp) } \theta_{1}\left\{\left(\frac{\theta_{1} \mathrm{t}_{4}^{4}}{8}-\frac{\theta_{1} \mathrm{t}_{3}^{4}}{8}+\frac{\mathrm{t}_{3}^{2}}{2}-\frac{\mathrm{t}_{4}^{2}}{2}\right)\left(\frac{\theta_{1} \mathrm{t}_{4}^{4}}{4}+\frac{\mathrm{t}_{4}^{2}}{2}\right)-\left(\frac{\theta_{1} \mathrm{t}_{4}^{6}}{24}-\frac{\theta_{1} \mathrm{t}_{3}^{6}}{24}+\frac{\theta_{1} \mathrm{t}_{3}^{6}}{48}-\frac{\theta_{1} \mathrm{t}_{4}^{6}}{48}-\frac{\theta_{1}^{2} \mathrm{t}_{1}^{8}}{64}+\frac{\theta_{1}^{2} \mathrm{t}_{4}^{8}}{64}+\frac{\mathrm{t}_{3}^{4}}{8}-\frac{\mathrm{t}_{4}^{4}}{8}\right)\right\}+(1- \\
& \mathrm{k})(\mathrm{a}-\mathrm{bp})\left[\left(\frac{\mathrm{t}_{6}^{3}}{8}+\frac{\mathrm{t}_{5}^{3}}{8}-\frac{\theta_{1} \mathrm{t}_{6}^{5}}{40}+\frac{\theta_{1} \mathrm{t}_{5}^{5}}{40}-\frac{\theta_{1}^{2} \mathrm{t}_{6}^{8}}{128}+\frac{\theta_{1}^{2} \mathrm{t}_{5}^{8}}{128}-\left(\frac{\mathrm{t}_{6}^{2}}{2}+\frac{\theta_{1} \mathrm{t}_{6}^{4}}{8}\right)\left(\mathrm{t}_{6}-\mathrm{t}_{5}-\frac{\theta_{1} \mathrm{t}_{6}^{3}}{6}+\frac{\theta_{1} \mathrm{t}_{5}^{3}}{6}\right)\right]+\mathrm{C}_{\mathrm{s}}(\mathrm{a}-\right. \\
& \text { bp) }\left\{\left(\frac{\mathrm{t}_{5}^{3}}{6}-\frac{\mathrm{t}_{4}^{3}}{6}-\frac{\mathrm{t}_{4}^{2}}{2}\left(\mathrm{t}_{5}-\mathrm{t}_{4}\right)\right)\right\}+\frac{\mathrm{G}_{\mathrm{D}}}{\mathrm{PS}}\left\{\frac{\mathrm{G}_{\mathrm{DF}}}{\mathrm{N}}+\mathrm{N} \cdot \mathrm{R}_{\mathrm{DV}} \cdot \prod_{\mathrm{j}=1}^{2} \mathrm{R}_{\mathrm{j}}\right\}
\end{aligned}
$$

\section{Algorithm to solve the mathematical model}

According to the proposed model, there are three independent variables in the total cost equation $t_{1}, t_{2}$ and $t_{5}$. To optimize the total cost equation and to find the value of all the independent parameters, the following steps are pursued.

Step. 1

Calculate the first-order partial derivatives w.r.t all the independent variable $\frac{\partial f}{\partial t_{1}}, \frac{\partial f}{\partial t_{2}}$ and $\frac{\partial f}{\partial t_{3}}$

Step 2.

Equate the first-order partial derivatives to zero and solve for the value of $t_{1}, t_{2}, t_{3}$

Step 3.

Now, calculate the second-order partial derivative w.r.t. all the independent variables like $\frac{\partial^{2} f}{\partial t_{1}^{2}}, \frac{\partial^{2} f}{\partial t_{1} \partial t_{2}}, \frac{\partial^{2} f}{\partial t_{1} \partial t_{3}}, \frac{\partial^{2} f}{\partial t_{2} \partial t_{1}}$ etc.

Step 4.

Now, form a Hessian matrix as follows

$$
\left(\begin{array}{ccc}
\frac{\partial^{2} f}{\partial t_{1}{ }^{2}} & \frac{\partial^{2} f}{\partial t_{1} \partial t_{2}} & \frac{\partial^{2} f}{\partial t_{1} \partial t_{3}} \\
\frac{\partial^{2} f}{\partial t_{2} \partial t_{1}} & \frac{\partial^{2} f}{\partial t_{2}{ }^{2}} & \frac{\partial^{2} f}{\partial t_{2} \partial t_{3}} \\
\frac{\partial^{2} f}{\partial t_{3} \partial t_{1}} & \frac{\partial^{2} f}{\partial t_{3} \partial t_{2}} & \frac{\partial^{2} f}{\partial t_{3}{ }^{2}}
\end{array}\right)
$$

Step 5. 
Find $\mathrm{H}_{1}, \mathrm{H}_{2}$, and $\mathrm{H}_{3}$, where, $\mathrm{H}_{1}, \mathrm{H}_{2}$, and $\mathrm{H}_{3}$ denote the first principal minor, second principal minor, and third principal minor respectively. If $\operatorname{det}\left(\mathrm{H}_{1}\right)>0$, $\operatorname{det}\left(\mathrm{H}_{2}\right)>0$, and $\operatorname{det}\left(\mathrm{H}_{3}\right)>0$, then the matrix is a positive definite matrix, and $\mathrm{f}$ is called convex function.

\section{Numerical analysis}

Two numerical examples are solved to show the reliability of the model. Random data is used to solve the following numerical.

\subsection{Numerical example 1}

Following parameters are used to demonstrate the numerical:

$\theta_{2}=\$ 0.5 ; \theta_{1}=\$ 0.9 ; \mathrm{w}=\$ 0.3 ; \mathrm{a}=44 ; \mathrm{b}=0.01 ; \mathrm{p}=\$ 100 ; c_{s}=\$ 0.01 ; D_{i}=\$ 0.001 ; \mathrm{k}=0.50 ; \mathrm{A}=\$ 6000 ; c_{r w}=\$ 0.13 ; c_{o w}=\$ 0.8 ; \mathrm{G}_{d}=\$ 5 ;$ $\mathrm{G}_{d v}=\$ 80 ; \mathrm{N}=70 ; \mathrm{R}_{D V}=\$ 0.1 ; \mathrm{r}_{1}=0.1 ; \mathrm{r}_{2}=0.9 ; \mathrm{PS}=\$ 4$;

By using the above parameters, we minimize the total cost function with green design and product stewardship, get the following values:

$\mathrm{TC}=\$ 5974.68 ; t_{1}=0.78214 ; t_{2}=1.22029 ; t_{5}=1.38124$;

Total cost and other parameter value without green design and product stewardship:

$\mathrm{TC}=\$ 5972.68 ; t_{1}=0.78214 ; t_{2}=1.22029 ; t_{5}=1.38124$;

\subsection{Sensitivity analysis for numerical 1}

Table 2. Sensitivity for parameter 'a'

\begin{tabular}{lllll}
\hline $\mathrm{a}$ & $\mathrm{t}_{1}$ & $\mathrm{t}_{2}$ & $\mathrm{t}_{5}$ & $\mathrm{TC}$ \\
\hline 44 & 0.78214 & 1.22029 & 1.38124 & 5974.68 \\
45 & 0.78163 & 1.22025 & 1.38136 & 5974.26 \\
46 & 0.78121 & 1.22028 & 1.38144 & 5973.62 \\
47 & 0.78075 & 1.22024 & 1.38155 & 5972.98 \\
\hline
\end{tabular}

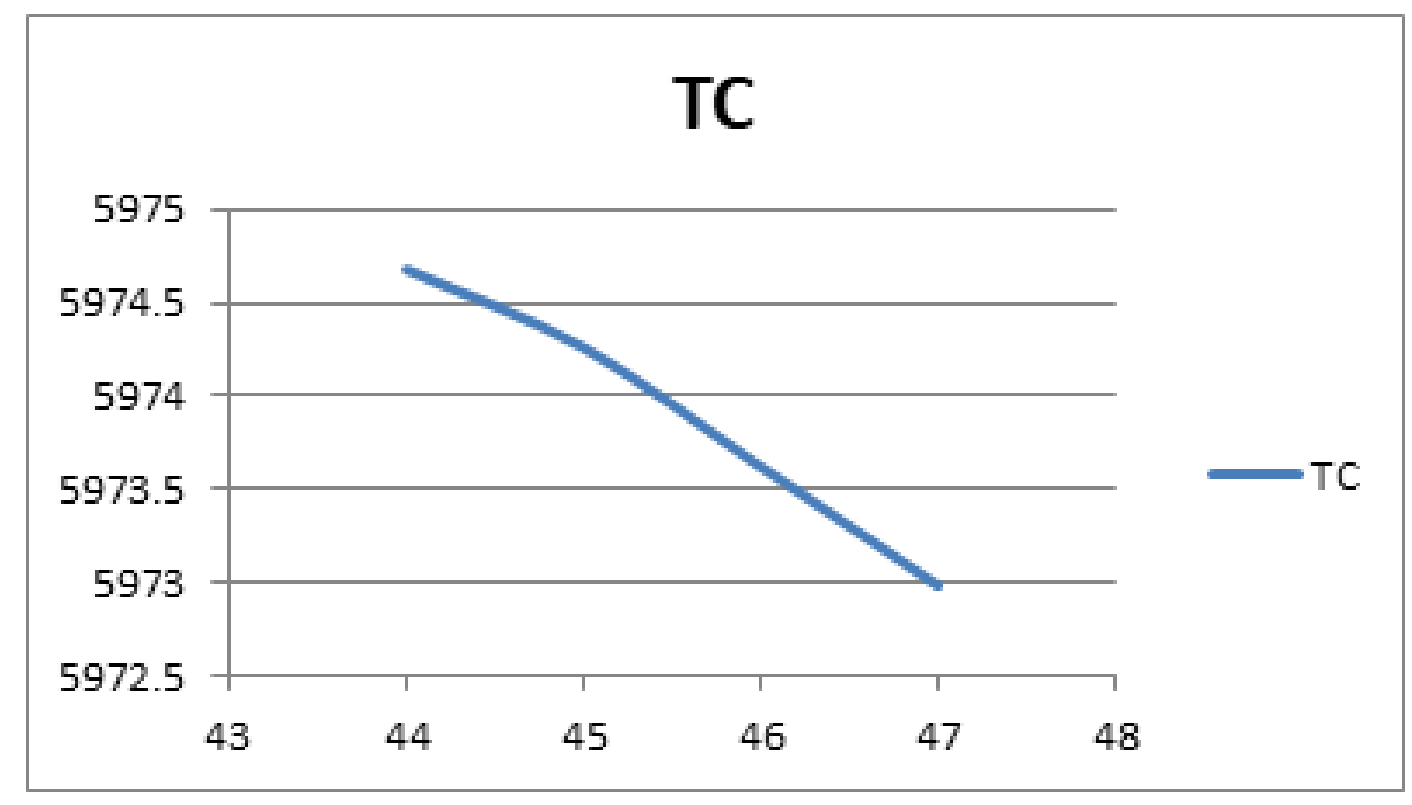

Fig 3. Sensitivity analysis between total cost and parameter 'a' 
Table 3. Sensitivity for the parameter ' $b$ '

\begin{tabular}{lllll}
\hline $\mathrm{b}$ & $\mathrm{t}_{1}$ & $\mathrm{t}_{2}$ & $\mathrm{t}_{5}$ & $\mathrm{TC}$ \\
\hline 0.008 & 0.782051 & 1.22029 & 1.38126 & 5974.77 \\
0.009 & 0.782083 & 1.22029 & 1.38126 & 5974.83 \\
0.010 & 0.782140 & 1.22029 & 1.38126 & 5974.89 \\
0.011 & 0.782150 & 1.22029 & 1.38126 & 5975.15 \\
\hline
\end{tabular}

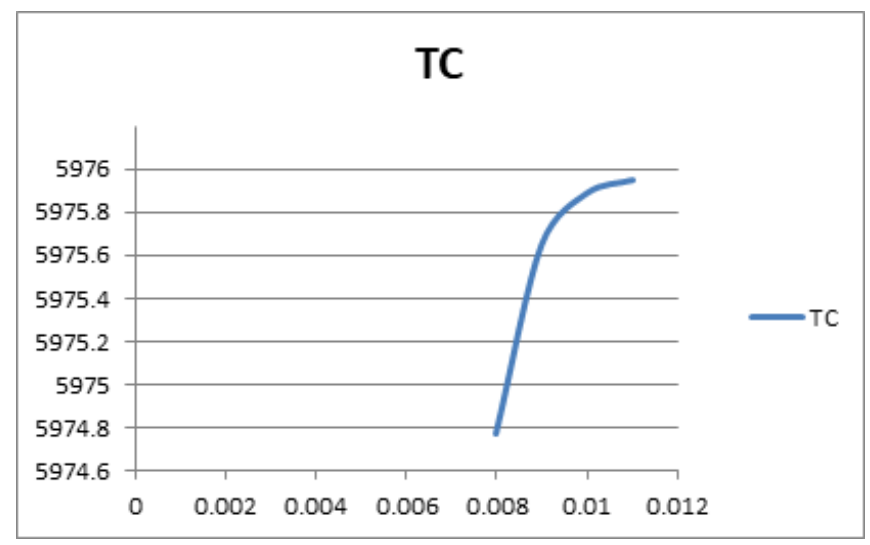

Fig 4. Sensitivity analysis between total cost and parameter 'b'

Table 4. Sensitivity for the parameter 'PS'

\begin{tabular}{lllll}
\hline PS & $\mathrm{t}_{1}$ & $\mathrm{t}_{2}$ & $\mathrm{t}_{5}$ & TC \\
\hline 4 & 0.78214 & 1.22029 & 1.38124 & 5974.68 \\
5 & 0.78214 & 1.22029 & 1.38124 & 5974.45 \\
6 & 0.78214 & 1.22029 & 1.38124 & 5975.05 \\
7 & 0.78214 & 1.22029 & 1.38124 & 5975.94 \\
\hline
\end{tabular}

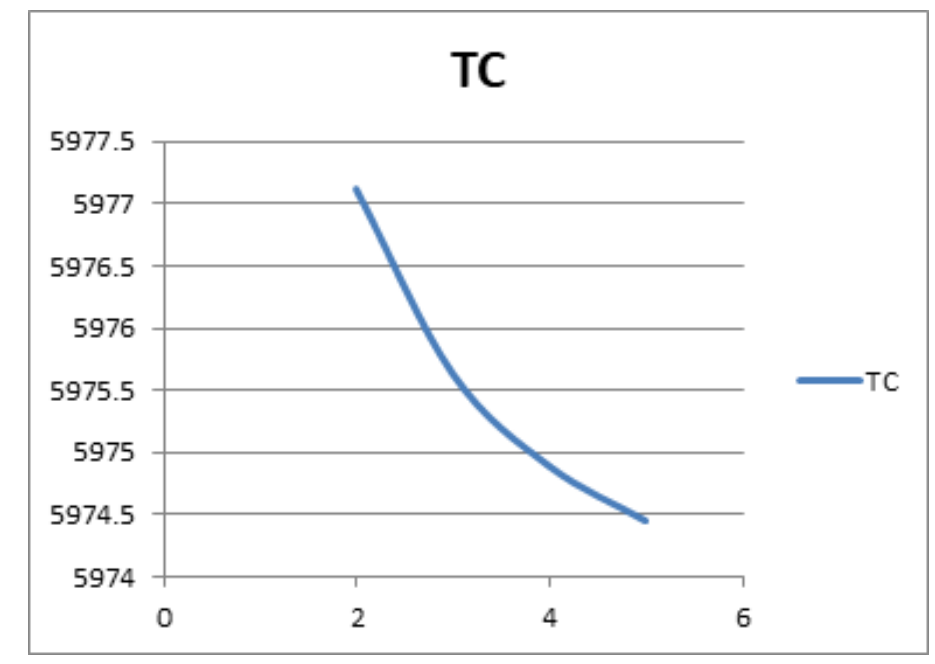

Fig 5. Sensitivity analysis between total cost and parameter 'PS' 
Table 5. Sensitivity for the parameter ' $\mathrm{G}_{d}$ '

\begin{tabular}{lllll}
\hline $\mathrm{G}_{d}$ & $\mathrm{t}_{1}$ & $\mathrm{t}_{2}$ & $\mathrm{t}_{5}$ & $\mathrm{TC}$ \\
\hline 5 & 0.78214 & 1.22029 & 1.38124 & 5974.68 \\
6 & 0.78214 & 1.22029 & 1.38124 & 5975.34 \\
7 & 0.78214 & 1.22029 & 1.38124 & 5975.78 \\
8 & 0.78214 & 1.22029 & 1.38124 & 5976.22 \\
\hline
\end{tabular}

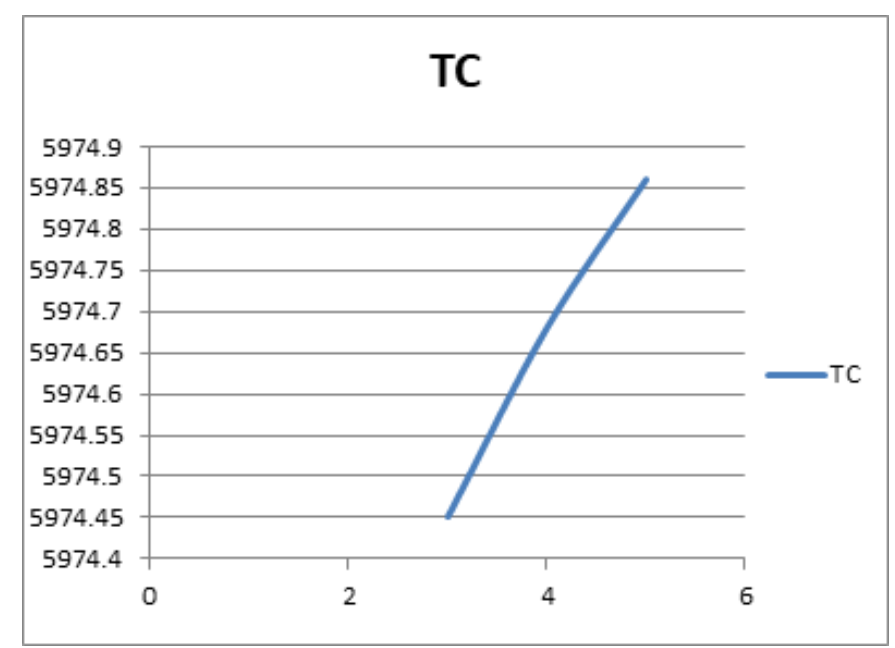

Fig 6. Sensitivity analysis between total cost and parameter ' $\mathrm{G}_{d}$ '

Table 6. Sensitivity for the parameter 'N'

\begin{tabular}{lllll}
\hline $\mathrm{N}$ & $\mathrm{t}_{1}$ & $\mathrm{t}_{2}$ & $\mathrm{t}_{5}$ & $\mathrm{TC}$ \\
\hline 60 & 0.78214 & 1.22029 & 1.38124 & 5975.02 \\
65 & 0.78214 & 1.22029 & 1.38124 & 5974.95 \\
70 & 0.78214 & 1.22029 & 1.38124 & 5974.89 \\
75 & 0.78214 & 1.22029 & 1.38124 & 5974.82 \\
\hline
\end{tabular}

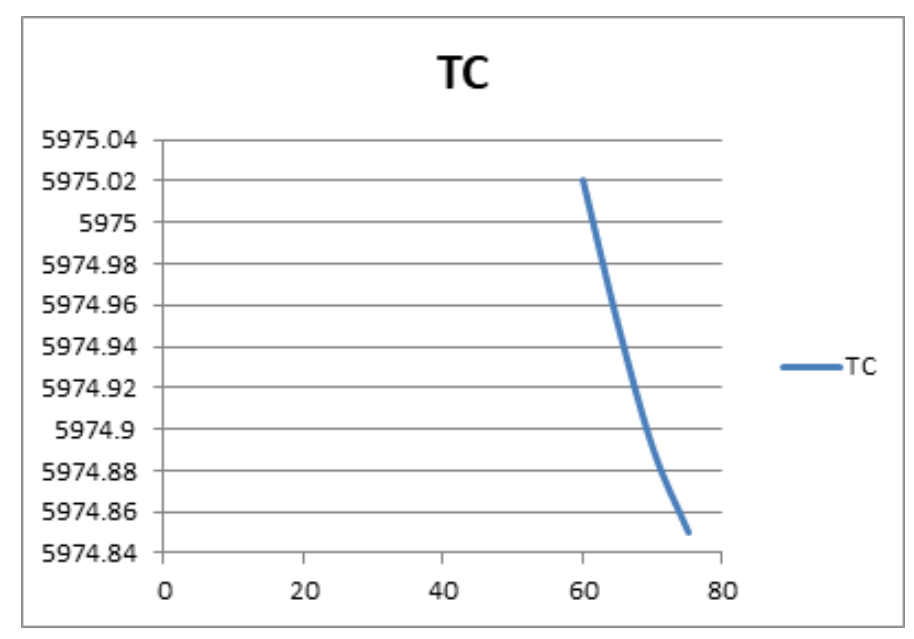

Fig 7. Sensitivity analysis between total cost and parameter 'N' 


\subsection{Observation for the above sensitivity analysis}

1. Firstly, when the value of 'a' increases then there is a decrease in the value of TC, but $t_{1}, t_{2}$ and $t_{5}$ becomes constant regularly. In any real market situation when demand increases then total cost decreases. Parameter 'a' is a factor of the demand function. Hence when the value of 'a' increases the value of demand function also increases, which may reduce total cost.

2. In the next step if we increase the value of ' $b$ ' then TC increases, but $t_{1}, t_{2}$ and $t_{5}$ will remain constant. Parameter ' $b$ ' is a negative impact on demand function. On increasing the value of ' $b$ ' demand decreases and if demand decrease then the total cost goes up.

3. On increasing the value of parameter 'PS' then TC increases regularly but, the value of $t_{5}, t_{1}$ and $t_{2}$ remains constant. Product stewardship is a concept which helps in making a product green. In the proposed research green design and product stewardship collaborated, by increasing the value of product stewardship, the total cost increases.

4. When the ' $\mathrm{G}_{d}$ ' increases then TC increases on the other hand $\mathrm{t}_{1}, \mathrm{t}_{2}$, and $\mathrm{t}_{5}$ remains constant. ' $\mathrm{G}_{d}$ ' is a green design life cycle cost if ' $\mathrm{G}_{d}$ ' increases then it also increases the value of total cost.

5. On increasing the number or recycling the product ' $N$ ' then the value of $t_{1}, t_{2}$, and $t_{5}$ remains constant, but the value of TC continuously reduces. It is the main objective of the paper that recycling of a commodity will reduce the total cost.

\subsection{Convexity of TC}

The Figures 8, 9 and 10 show the convexity of TC function w.r.t different independent variables.

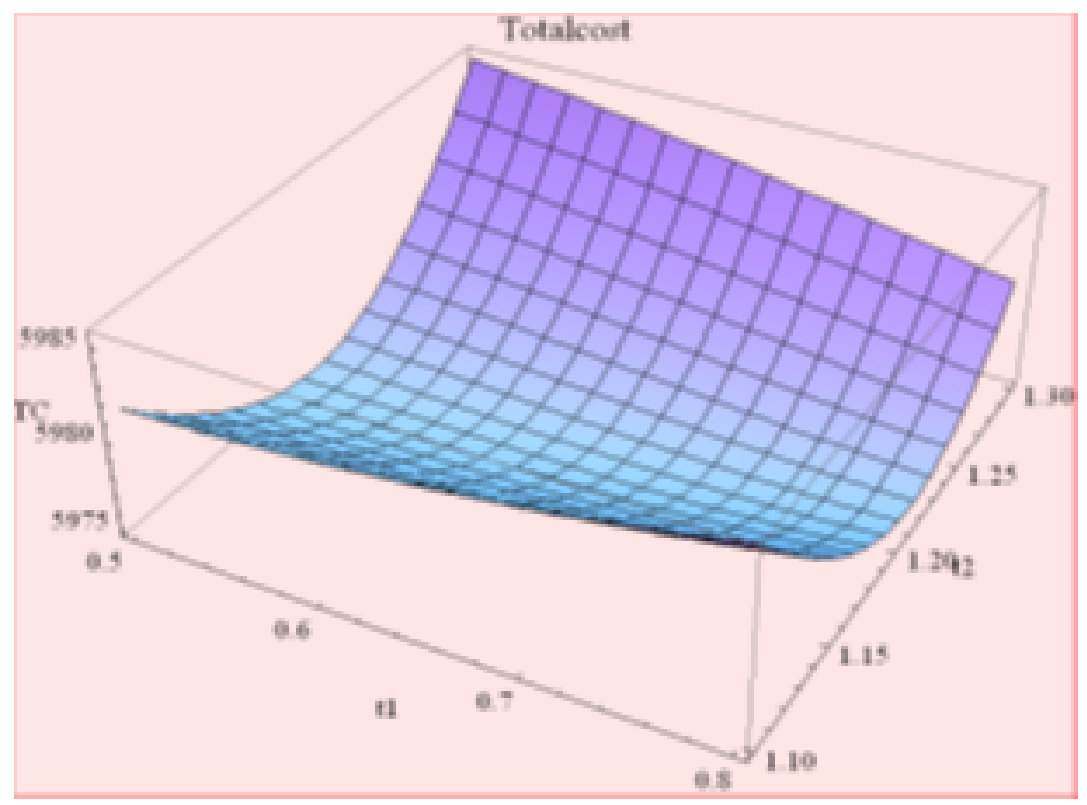

Fig 8. TC w.r.t $t_{1}$ and $t_{2}$ 


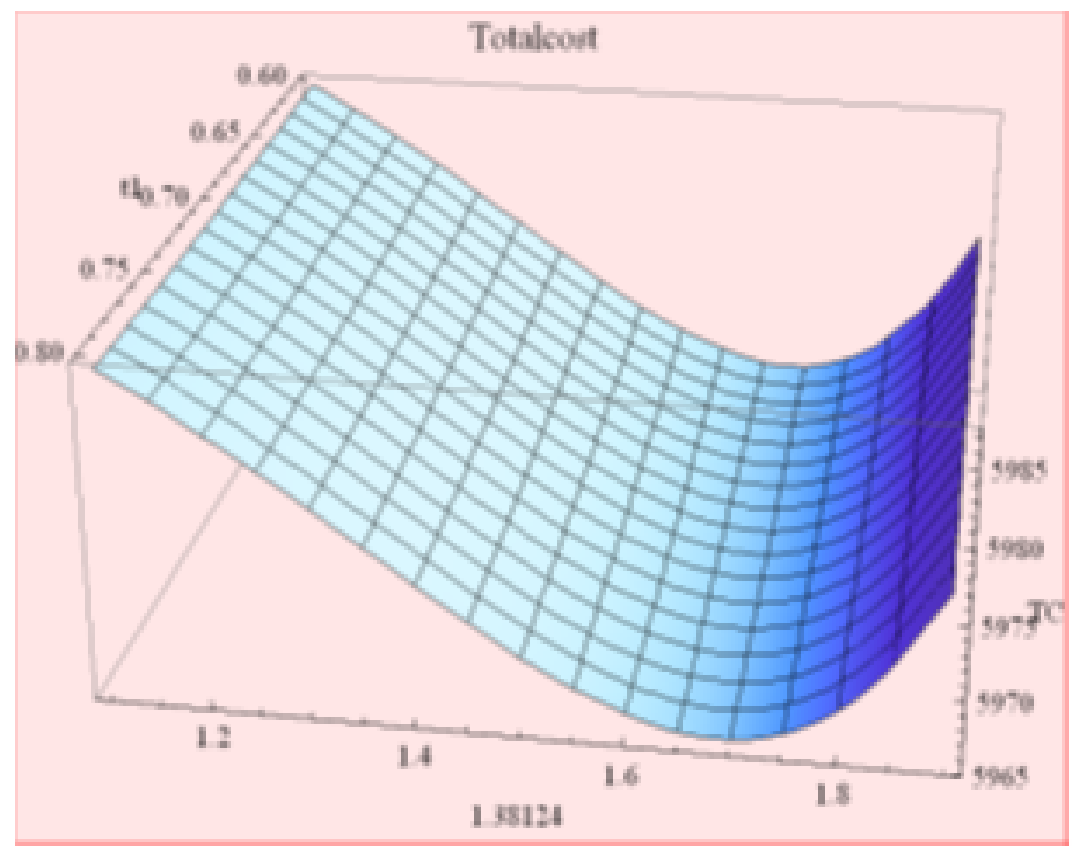

Fig 9. TC w.r.t $t_{1}$ and $t_{5}$

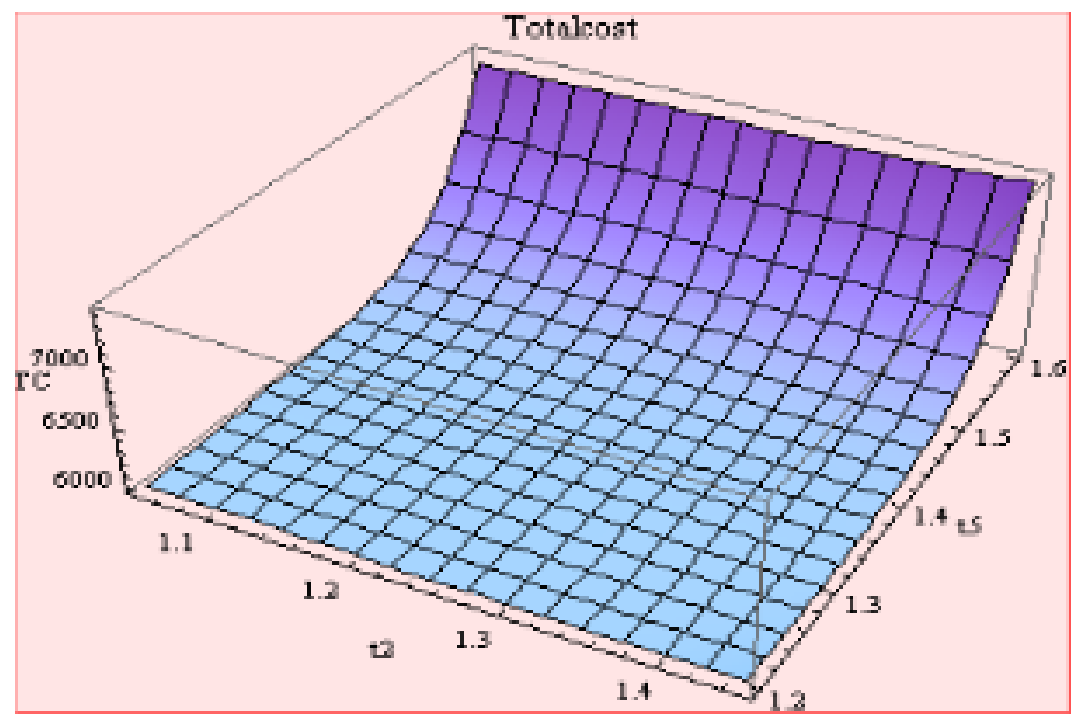

Fig 10. TCw.r.t $t_{2}$ and $t_{5}$

\subsection{Numerical example 2}

Following parameters are used to demonstrate the numerical:

$\theta_{2}=\$ 0.1 ; \theta_{1}=\$ 0.5 ; \mathrm{w}=\$ 0.1 ; \mathrm{a}=40 ; \mathrm{b}=0.001 ; \mathrm{p}=\$ 90 ; c_{s}=\$ 0.01 ; D_{i}=\$ 0.004 ; \mathrm{k}=0.40 ; \mathrm{A}=\$ 6000 ; c_{r w}=\$ 0.09 ; c_{o w}=\$ 0.6 ; \mathrm{G}_{d}=\$ 5 ;$ $\mathrm{G}_{d v}=\$ 75 ; \mathrm{N}=40 ; \mathrm{R}_{D V}=\$ 0.01 ; \mathrm{r}_{1}=0.01 ; \mathrm{r}_{2}=0.7 ; \mathrm{PS}=\$ 3 ;$

By using above parameters, we minimize the total cost function and get the following values:

$\mathrm{TC}=\$ 5919.74 ; t_{1}=0.7 .334 ; t_{2}=0.831332 ; t_{5}=2.09251$; 


\subsection{Sensitivity analysis for above numerical}

Table 7. Sensitivity for the parameter 'a'

\begin{tabular}{lllll}
\hline $\mathrm{a}$ & $\mathrm{t}_{1}$ & $\mathrm{t}_{2}$ & $\mathrm{t}_{5}$ & $\mathrm{TC}$ \\
\hline 20 & 0.712615 & 0.83245 & 2.09258 & 5961.51 \\
30 & 0.708811 & 0.835243 & 2.09256 & 5940.60 \\
40 & 0.70334 & 0.831332 & 2.09251 & 5919.74 \\
50 & 0.70225 & 0.832285 & 2.09252 & 5898.85 \\
\hline
\end{tabular}

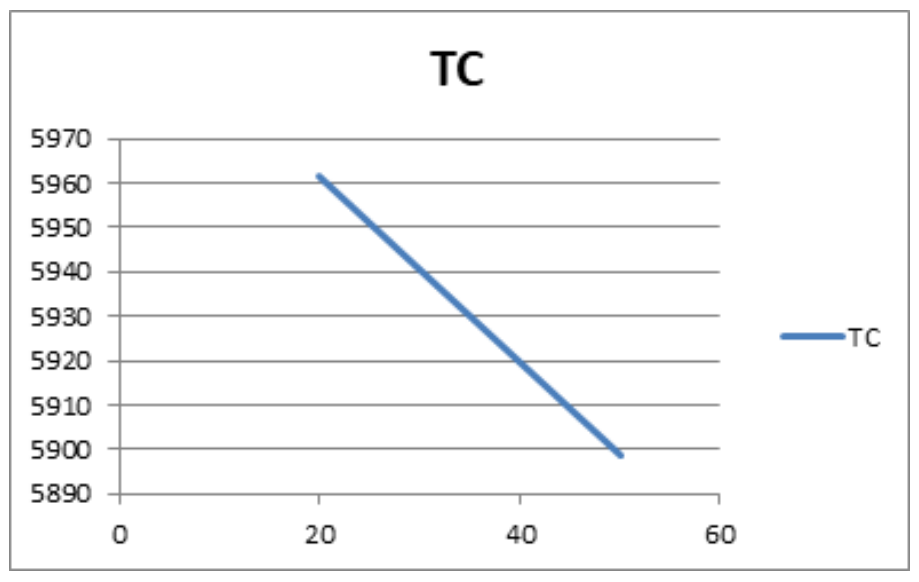

Fig 11. Sensitivity analysis between total cost and parameter 'a'

Table 8. Sensitivity for the parameter 'b'

\begin{tabular}{lllll}
\hline $\mathrm{b}$ & $\mathrm{t}_{1}$ & $\mathrm{t}_{2}$ & $\mathrm{t}_{5}$ & $\mathrm{TC}$ \\
\hline 0.0005 & 0.703323 & 0.831330 & 2.09251 & 5919.65 \\
0.0010 & 0.70334 & 0.831332 & 2.09251 & 5919.74 \\
0.0015 & 0.703342 & 0.8331329 & 2.09251 & 5919.84 \\
0.0020 & 0.703358 & 0.831338 & 2.09251 & 5919.93 \\
\hline
\end{tabular}

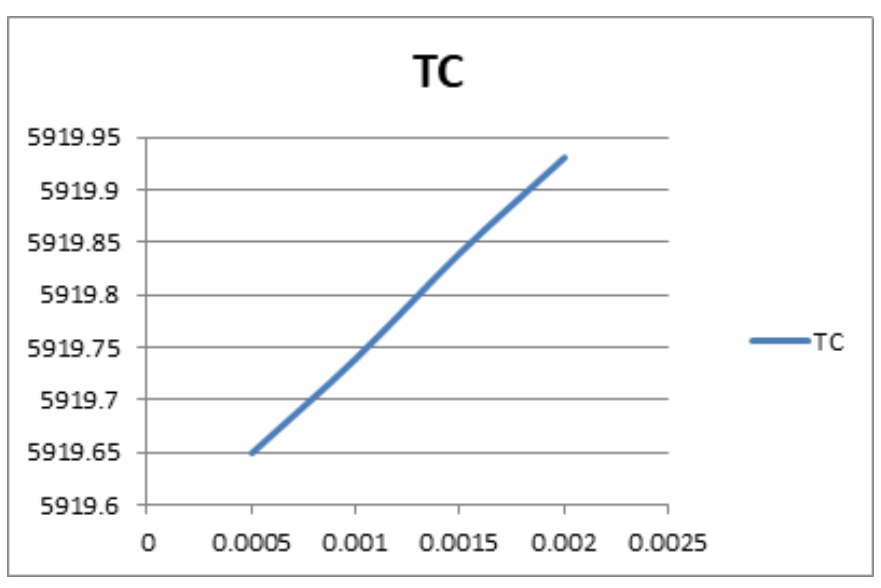

Fig 12. Sensitivity analysis between total cost and parameter 'b' 
Table 9. Sensitivity for the parameter 'PS'

\begin{tabular}{lllll}
\hline PS & $\mathrm{t}_{1}$ & $\mathrm{t}_{2}$ & $\mathrm{t}_{5}$ & $\mathrm{TC}$ \\
\hline 1 & 0.70334 & 0.831332 & 2.09251 & 5926.00 \\
2 & 0.703334 & 0.831332 & 2.09251 & 5921.31 \\
3 & 0.703334 & 0.831332 & 2.09251 & 5919.74 \\
4 & 0.7 .3334 & 0.831332 & 2.09251 & 5918.96 \\
\hline
\end{tabular}

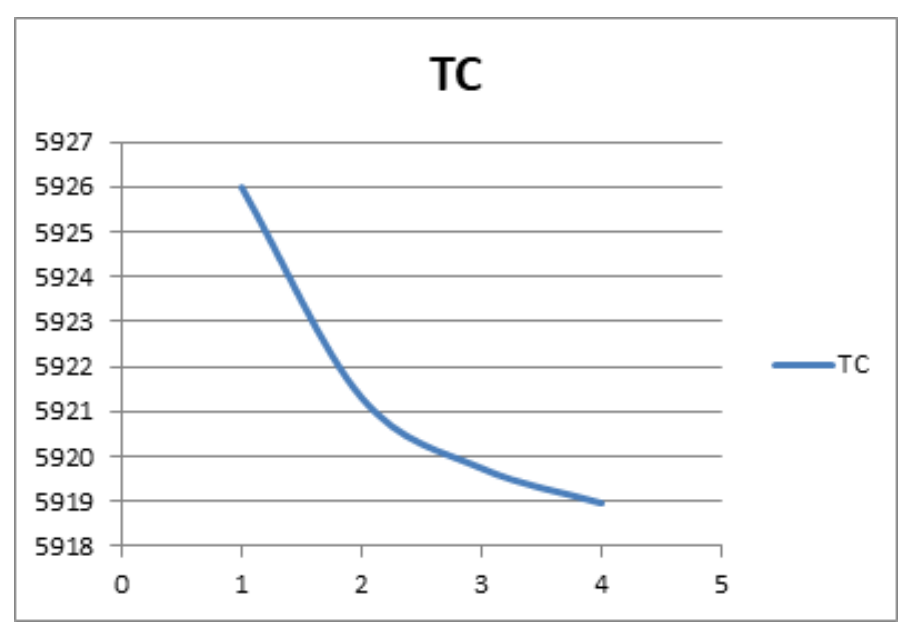

Fig 13. Sensitivity analysis between total cost and parameter 'PS'

Table 10. Sensitivity for the parameter ' $\mathrm{G}_{d}$ '

\begin{tabular}{lllll}
\hline $\mathrm{G}_{d}$ & $\mathrm{t}_{1}$ & $\mathrm{t}_{2}$ & $\mathrm{t}_{5}$ & $\mathrm{TC}$ \\
\hline 3 & 0.7 .3334 & 0.831332 & 2.09251 & 5918.49 \\
4 & 0.703334 & 0.831332 & 2.09251 & 5919.12 \\
5 & 0.703334 & 0.831332 & 2.09251 & 5919.74 \\
6 & 0.7 .3334 & 0.831332 & 2.09251 & 5920.37 \\
\hline
\end{tabular}

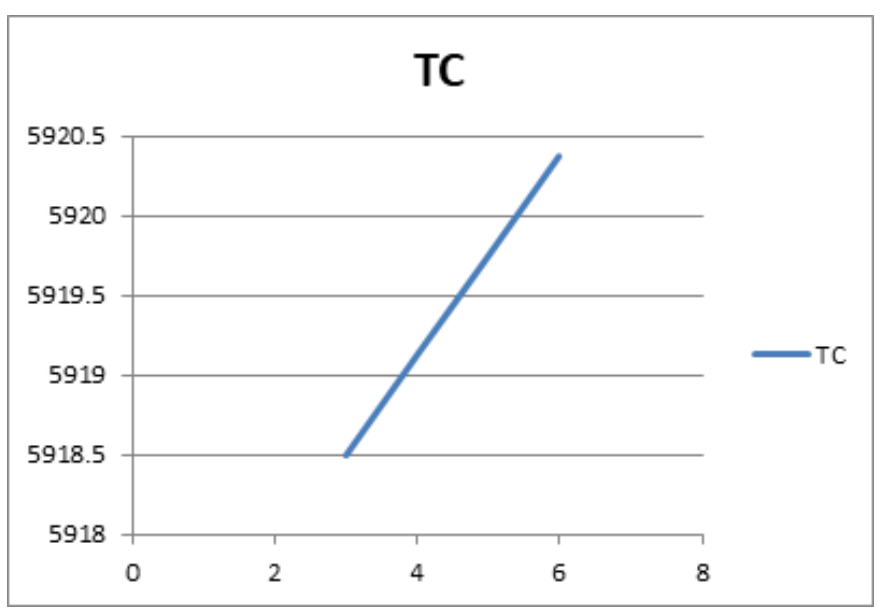

Fig 14. Sensitivity analysis between total cost and parameter ' $\mathrm{G}_{d}$ ' 
Table 11. Sensitivity for the parameter 'N'

\begin{tabular}{lllll}
\hline $\mathrm{N}$ & $\mathrm{t} 1$ & $\mathrm{t} 2$ & $\mathrm{t} 5$ & $\mathrm{TC}$ \\
\hline 20 & 0.703334 & 0.831332 & 2.09251 & 5922.87 \\
30 & 0.703334 & 0.831332 & 2.09251 & 5920.78 \\
40 & 0.703334 & 0.831332 & 2.09251 & 5919.74 \\
50 & 0.73334 & 0.831332 & 2.09251 & 5919.12 \\
\hline
\end{tabular}

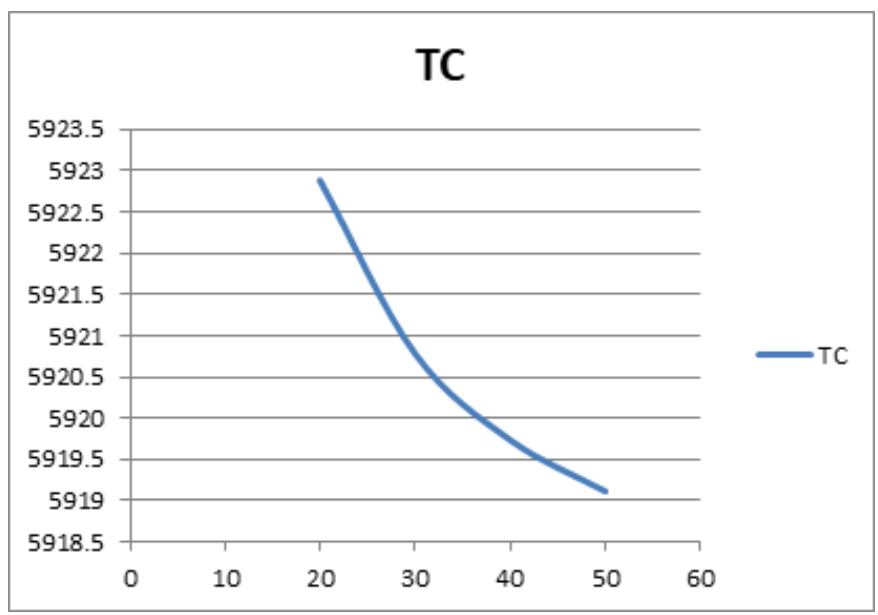

Fig 15. Sensitivity analysis between total cost and parameter 'N'

\subsection{Observation for the above sensitivity analysis:}

1. Firstly, when the value of 'a' increases then there is decrease in the value of TC and $t_{1}$, but on the other side the values of $t_{2}$ fluctuate regularly. The value of $t_{5}$ increases first and after that decreases. On increasing the value of parameter ' $a$ ' demand function is also increasing. In any situation when demand goes up, it will decrease the total cost.

2. In the next step if the value of ' $b$ ' increased then TC increases, also increase, and $t_{1}, t_{5}$ and $t_{2}$ will remain constant. On increasing the value of parameter ' $b$ ' demand function decreases and when demand decrease then total cost increase.

3. On increasing the value of parameter 'PS'(product stewardship), TC decreased. On the other hand $t_{1}, t_{5}$ and $t_{2}$ become constant. Product stewardship has a sharp effect on green product design. If the parameter of product stewardship increases then total cost decreased.

4. When ' $\mathrm{G}_{d}$ ' increase then TC increased, but the value oft ${ }_{2}, \mathrm{t}_{5}$, and $\mathrm{t}_{1}$ will become constant. When green design cycle cost increases then green product design cost also increases, it will increase the total cost also..

5. $\mathrm{n}$ increasing the number of recycling the product ' $\mathrm{N}$ ' then the value of $\mathrm{t} 1, \mathrm{t} 2,{ }_{a} \mathrm{nd} \mathrm{t} 5$ remains constant, but the value of TC continuously reduces.

\subsection{Convexity of TC}

In the following Figures 16, 17 and 18 shows the convexity of TC function w.r.t independent variables. 


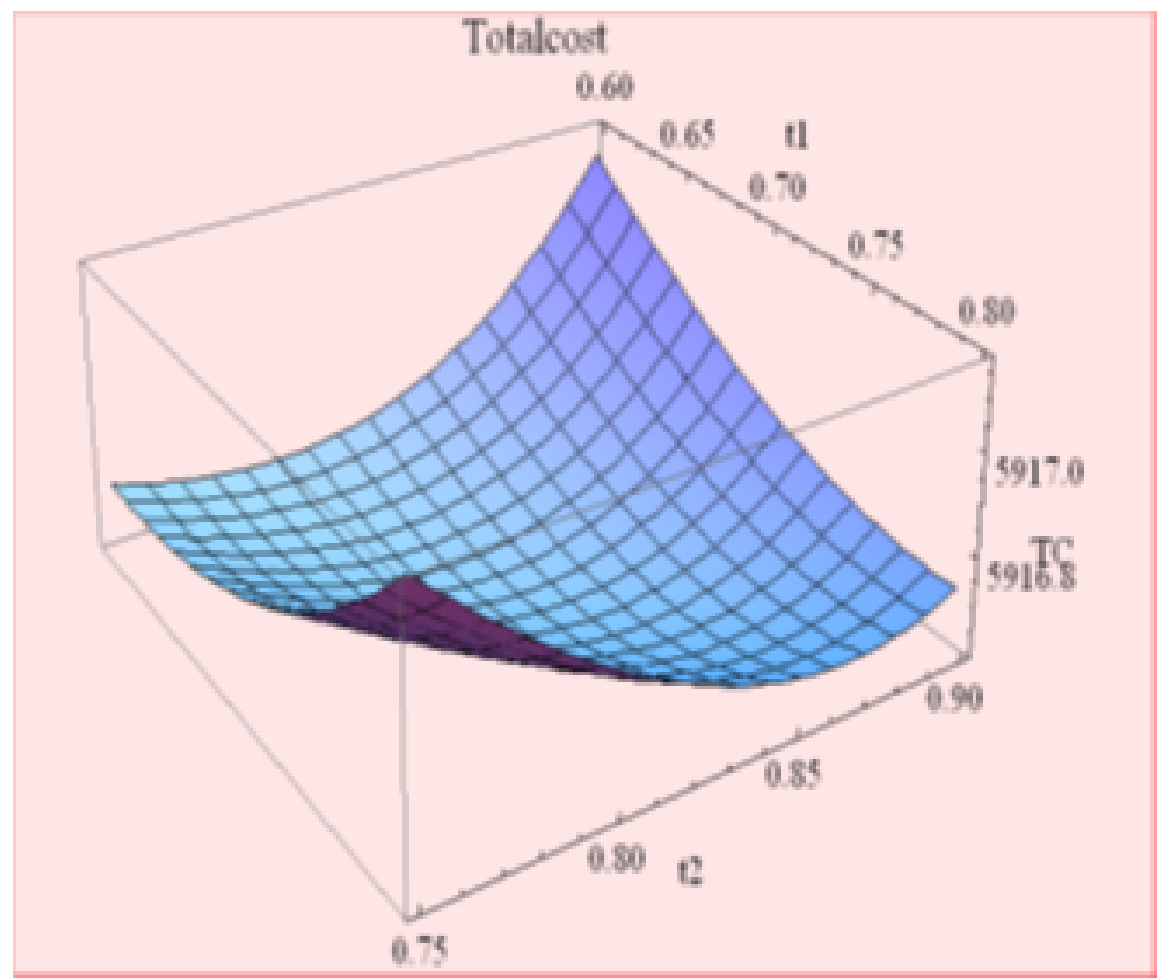

Fig 16. TCw.r.t $t_{1}$ and $t_{2}$

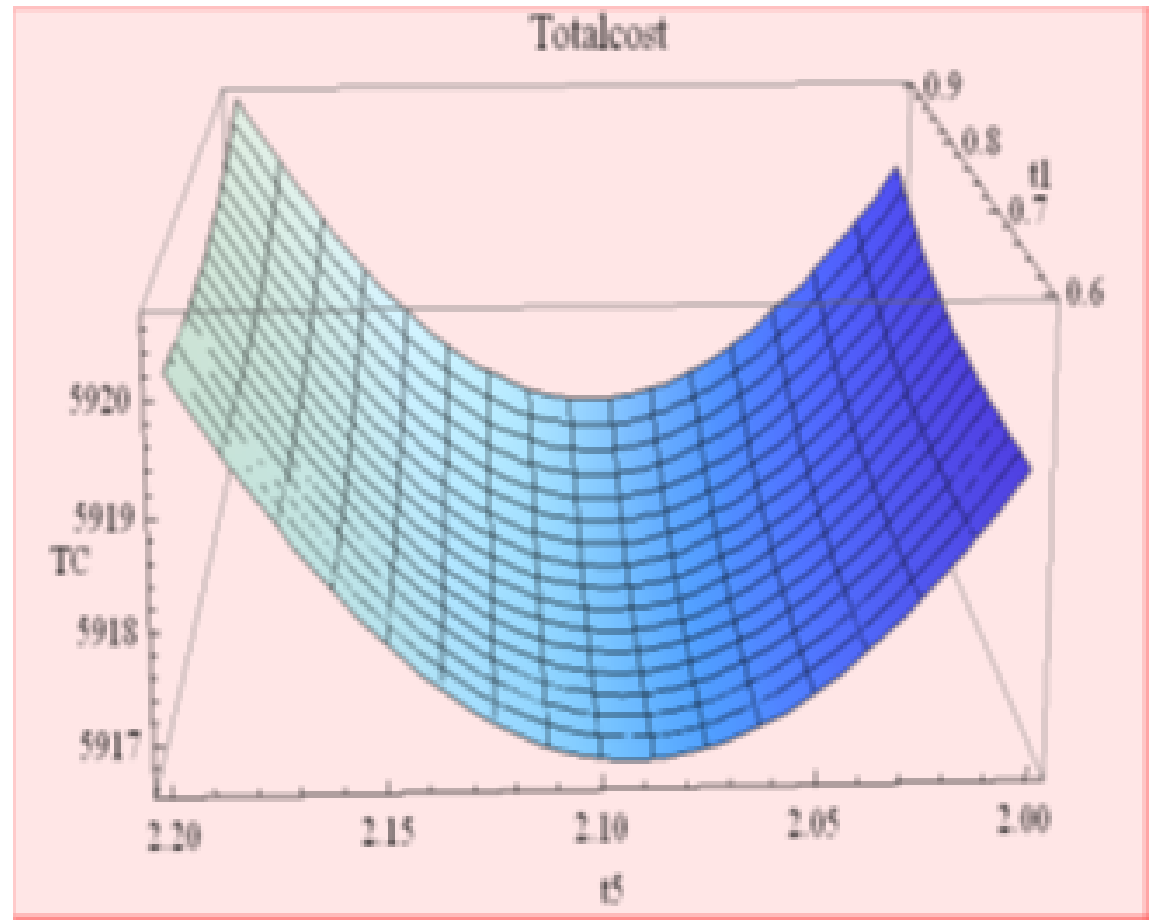

Fig 17. TCw.r.t $t_{1}$ and $t_{5}$ 


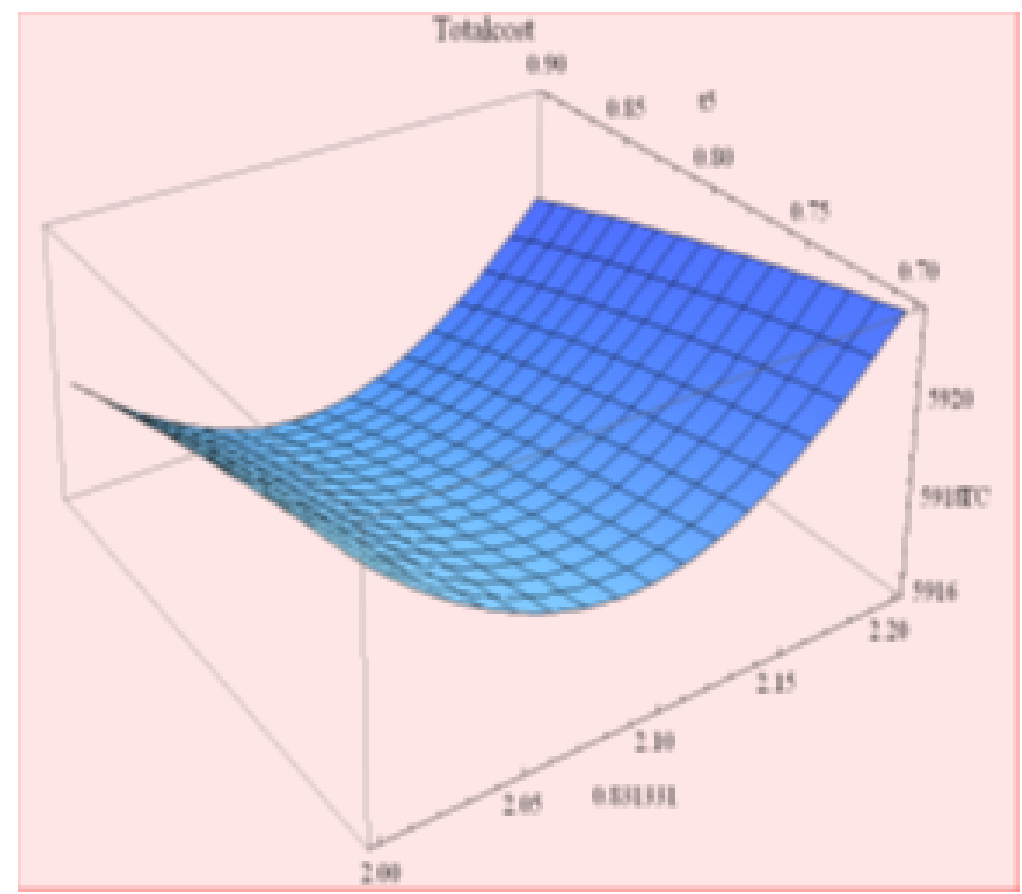

Fig 18. TC w.r.t $\mathrm{t}_{2}$ and $\mathrm{t}_{5}$

\section{Conclusion}

This study has collaborated on the concepts of green design, product stewardship, and two warehouses with the recycling of the used items. Numerical examples and sensitivity analysis illustrate that number of recycling of the items had a reverse effect on the total cost. i.e., an increase in the number of recycles results in the reduction of the total cost. Green design and product stewardship help the manufacturers to decrease the recycling cost. This study may be very useful for the manufacturer to deal with inventory management as well as environmental issues. Future research can be unfolded as integral research model by which of inflation, trade credit policy, different demand patterns, etc. can be included.

\section{References}

1) Frosch AR, Gallopoulos EN. Strategies for Manufacturing. Scientific American. 1989;261(3):144-152. Available from: https://dx.doi.org/10.1038/ scientificamerican0989-144.

2) Glantschnig WJ. Green design: an introduction to issues and challenges. IEEE Transactions on Components, Packaging, and Manufacturing Technology: Part A. 1994;17(4):508-513. Available from: https://dx.doi.org/10.1109/95.335033.

3) Shapiro G, White AL. Right from the start: Product stewardship through life cycle design. Corporate Environmental Strategy. 1999;6(1):80003-80005. Available from: https://doi.org/10.1016/S1066-7938(00)80003-2.

4) Bellmann K, Khare A. Economic issues in recycling end-of-life vehicles. Technovation. 2000;20:677-690. Available from: https://dx.doi.org/10.1016/ s0166-4972(00)00012-2.

5) Das JK. Responding to green concern: the role for government and business. Vikalpa. 2002;27:3-12. Available from: https://doi.org/10.1177/ 0256090920020102.

6) Lewis M. Defining product stewardship and sustainability in the Australian packing industry. Environmental Science \& Policy. 2005;8(1):45-55. Available from: https://dx.doi.org/10.1016/j.envsci.2004.09.002.

7) Peck EJ, Christy AJ. Putting the stewardship concept into practice: Commercial moss harvest in Northwestern Oregon, USA. Forest Ecology and Management. 2006;225(1-3):225-233. Available from: https://dx.doi.org/10.1016/j.foreco.2005.12.054.

8) Burger J. Environmental management: Integrating ecological evaluation, remediation, restoration, natural resources damage assessment and long term stewardship on contaminated lands. Science of the Total Environment. 2008;400(1-3):6-19. Available from: https://doi.org/10.1016/j.scitotenv.2008.06.041.

9) Chung CJ, Wee HM. Green-component life cycle value on design and reverse manufacturing in semi-closed supply chain. International Journal of Production Economics. 2008;113(2):528-545. Available from: https://doi.org/10.1016/j.ijpe.2007.10.020.

10) Shi VG, Koh SCL, Baldwin J, Cucchiella F. Natural resource based green supply chain management. Supply Chain Management: An International Journal. 2012;17(1):54-67. Available from: https://dx.doi.org/10.1108/13598541211212203.

11) Wong WYC, hung Lai K, Shang KC, Lu CS, Leung TKP. Green operations and the moderating role of environmental management capability of suppliers on manufacturing firm performance. International Journal of Production Economics. 2012;140(1):283-294. Available from: https://dx.doi.org/10.1016/j. ijpe.2011.08.031. 
12) Zhou Y. A multi ware house inventory model for deteriorating items with time varying demand and shortages. Journal of Computer and Operation Research. 2003;30:2115-2134. Available from: https://doi.org/10.1016/S0305-0548(02)00126-0.

13) Mallidis I, Vlachos D, Iakovou E, Dekker R. Design and planning for green global supply chains under periodic review replenishment policies. Transportation Research Part E: Logistics and Transportation Review. 2014;72:210-235. Available from: https://dx.doi.org/10.1016/j.tre.2014.10.008.

14) Domingo L, Rio M. Linking Use Stage Life Cycle Inventories with Product Design Models of Usage. Procedia CIRP. 2016;48:342-347. Available from: https://dx.doi.org/10.1016/j.procir.2016.03.149.

15) Zhu W, He Y. Green product design in supply chain under competition. European Journal of Operational Research. 2017;258(1):165-180. Available from: https://doi.org/10.1016/j.ejor.2016.08.053.

16) Balakrishnan AS, Suresh J. Green supply chain management in Indian automotive sector. International Journal of logistics System and Management. 2018;29(4):502-523. Available from: https://doi.org/10.1504/IJLSM.2018.090476.

17) Yadav AS, Johri M, Singh J, Uppal S. Analysis of green supply chain inventory management for warehouse with environmental collaboration and sustainability performance using genetic algorithm. International Journal of Pure and Applied Mathematics. 2018;118(20):155-161. Available from: https://acadpubl.eu/jsi/2018-118-20/articles/20a/21.pdf.

18) Khalafi S, Zarei M. Design of a green supply chain network by considering the possibility of storage and location-routing problem under certainty. International Journal of logistics System and Management. 2019;34(2):253-268. Available from: https://doi.org/10.1504/IJLSM.2019.102216.

19) Huo B, Gu M, Wang Z. Green or lean? A supply chain approach to sustainable performance. Journal of Cleaner Production. 2019;216:152-166. Available from: https://dx.doi.org/10.1016/j.jclepro.2019.01.141.

20) Du P, Yang X, Xu L, Tan Y, Li H. Green design strategies of competing manufacturers in a sustainable supply chain. Journal of Cleaner Production. $2020 ; 265$. Available from: https://dx.doi.org/10.1016/j.jclepro.2020.121853.

21) Hartely R. A management emphasis. Good Year, CA. In: and others, editor. Operation Research. 1976;p. 182-192.

22) Sarma K. A deterministic order level inventory model with two level of storage and an optimum release rule. Opsearch. 1983;20:175-180. Available from: https://doi.org/10.1016/0377-2217(87)90194-9.

23) Dave U. EOQ model with two level storage. Opsearch. 1988;25:190-196.

24) Kar S, Bhunia AK, Maiti M. Deterministic inventory model with two levels of storage, a linear trend in demand and a fixed time horizon. Computers \& Operations Research. 2001;28(13):1315-1331. Available from: https://dx.doi.org/10.1016/s0305-0548(00)00042-3.

25) Zhou M, Dun Y, Chen Z, Yang W. Optimizing green production strategies: An integrated approach. Computer and Industrial Engineering. 2013;65(3):517528. Available from: https://doi.org/10.1016/j.cie.2013.02.020.

26) Yang HL. Two ware house Partial backlogging inventory model for deteriorating items under inflation. International Journal of Production Economics. 2006;103(1):362-370. Available from: https://doi.org/10.1016/j.jpe.2005.09.003.

27) Singh SR, Singh S. Two ware house partial backlogging inventory model for perishable product having exponential demand. International Journal of Mathematical Sciences \& Computer. 2008;1(1):229-236. Available from: http://acadpubl.eu/hubSpecialIssue.

28) Lee C, Hsu S. A two ware house production model for deteriorating inventory items with time dependent demand. European Journal of Operational Research. 2009;194:700-710. Available from: https://doi.org/10.1016/j.ejor.2007.12.034.

29) Singh SR, Kumar N, Kumari R. An inventory model for deteriorating items with shortages and stock-dependent demand under inflation for two-shops under one management. OPSEARCH. 2010;47(4):311-329. Available from: https://dx.doi.org/10.1007/s12597-010-0026-x.

30) Hariga M. Inventory model for multi ware house under fixed and flexible space leasing contract. Computers and Industrial Engineering. 2012;61:744-751. Available from: https://doi.org/10.1016/j.cie.2011.05.006.

31) Sett BK, Sarkar B, \&amp;goswami A. A two ware house inventory model with increasing demand and time dependent deterioration rate. ScientiaIranica. 2012;19(6):1969-1977. Available from: https://doi.org/10.1016/j.scient.2012.10.040.

32) Singh C, Singh SR. Optimal ordering policy for deteriorating items with power-form stock dependent demand under two-warehouse storage facility. OPSEARCH. 2013;50(2):182-196. Available from: https://dx.doi.org/10.1007/s12597-012-0105-2.

33) Kumar N, Singh S, Kumari R. A two ware house inventory model for deteriorating items with three component demand rate and time-proportional backlogging rate and fuzzy environment. International Journal of industrial Engineering Computation. 2013;4(4):587-598. Available from: https: //doi.org/10.1016/j.protcy.2013.12.423.

34) Khurana D. Two ware house inventory model for deteriorating items with time dependent demand under inflation. International Journal of Computer Applications. 2015;114(7):34-38. Available from: https://doi.org/10.5120/19994-1738.

35) Singh SR, Rathoreh. A two ware house inventory model with non-instantaneous deterioration and partial backlogging. Seventh International Conference on Contemporary Computing. 2014;p. 431-436. Available from: https://doi.org/10.1109/IC3.2014.6897212. 